\title{
A New Synthetic Route to Authentic $N$-Substituted Aminomaleimides
}

\author{
N. R. Conley, R. J. Hung, and C. G. Willson* \\ The University of Texas at Austin, Department of Chemistry and Biochemistry, 1 \\ University Station, A5300, Austin, TX 78712-0164, USA
}

\section{Content of Supporting Information}

(34 pages)

Page S-1: Title of the paper, authors' names, and address along with the contents

Page S-2: ${ }^{1} \mathrm{H}$ NMR spectrum of compound $\mathbf{1 b}$

Page S-3: ${ }^{1} \mathrm{H}$ NMR spectra $(\delta 6.0-\delta 8.4)$ of compound $\mathbf{1 b}$ at $130{ }^{\circ} \mathrm{C}, 90{ }^{\circ} \mathrm{C}$, and $27{ }^{\circ} \mathrm{C}$

Page S-4: ${ }^{1} \mathrm{H}$ NMR NOE/exchange spectra for compound $\mathbf{1 b}$

Page S-5: ${ }^{13} \mathrm{C}$ NMR spectrum of compound $\mathbf{1 b}$

Page S-6: IR (KBr) spectrum of compound $\mathbf{1 b}$

Page S-7: ${ }^{1} \mathrm{H}$ NMR spectrum of compound $\mathbf{3 b}$

Page S-8: ${ }^{13} \mathrm{C} /{ }^{1} \mathrm{H}$ NMR correlation spectrum of compound $\mathbf{3 b}$

Page S-9: Long range ${ }^{13} \mathrm{C} /{ }^{1} \mathrm{H}$ NMR correlation spectrum of compound $\mathbf{3 b}$

Page S-10: ${ }^{13} \mathrm{C}$ NMR spectrum of compound $\mathbf{3 b}$

Page S-11: IR (KBr) spectrum of compound $\mathbf{3 b}$

Page S-12: Crystal structure of compound $\mathbf{3 b}$

Page S-13: ${ }^{1} \mathrm{H}$ NMR spectrum of compound 9b

Page S-14: ${ }^{13} \mathrm{C}$ NMR spectrum of compound $9 \mathbf{b}$

Page S-15: IR (KBr) spectrum of compound 9b

Page S-16: ${ }^{1} \mathrm{H}$ NMR spectrum of compound 10b

Page S-17: ${ }^{13} \mathrm{C}$ NMR spectrum of compound $\mathbf{1 0 b}$

Page S-18: IR (KBr) spectrum of compound $\mathbf{1 0 b}$

Page S-19: Crystal structure of compound 10b

Page S-20: ${ }^{1} \mathrm{H}$ NMR spectrum of compound $\mathbf{2 b}$

Page S-21: ${ }^{13} \mathrm{C}$ NMR spectrum of compound $\mathbf{2 b}$

Page S-22: IR (KBr) spectrum of compound $2 \mathbf{b}$

Page S-23: Crystal structure of compound $\mathbf{2 b}$

Page S-24: ${ }^{1} \mathrm{H}$ NMR spectrum of compound 9c

Page S-25: ${ }^{13} \mathrm{C}$ NMR spectrum of compound $9 \mathrm{c}$

Page S-26: IR (KBr) spectrum of compound 9c

Page S-27: ${ }^{1} \mathrm{H}$ NMR spectrum of compound 10c

Page S-28: ${ }^{13} \mathrm{C}$ NMR spectrum of compound 10c

Page S-29: IR (KBr) spectrum of compound 10c

Page S-30: ${ }^{1} \mathrm{H}$ NMR spectrum of compound 10d

Page S-31: ${ }^{13} \mathrm{C}$ NMR spectrum of compound 10d

Page S-32: IR (KBr) spectrum of compound 10d

Page S-33: ${ }^{1} \mathrm{H}$ NMR spectrum of compound 3d

Page S-34: ${ }^{13}$ C NMR spectrum of compound 3c 


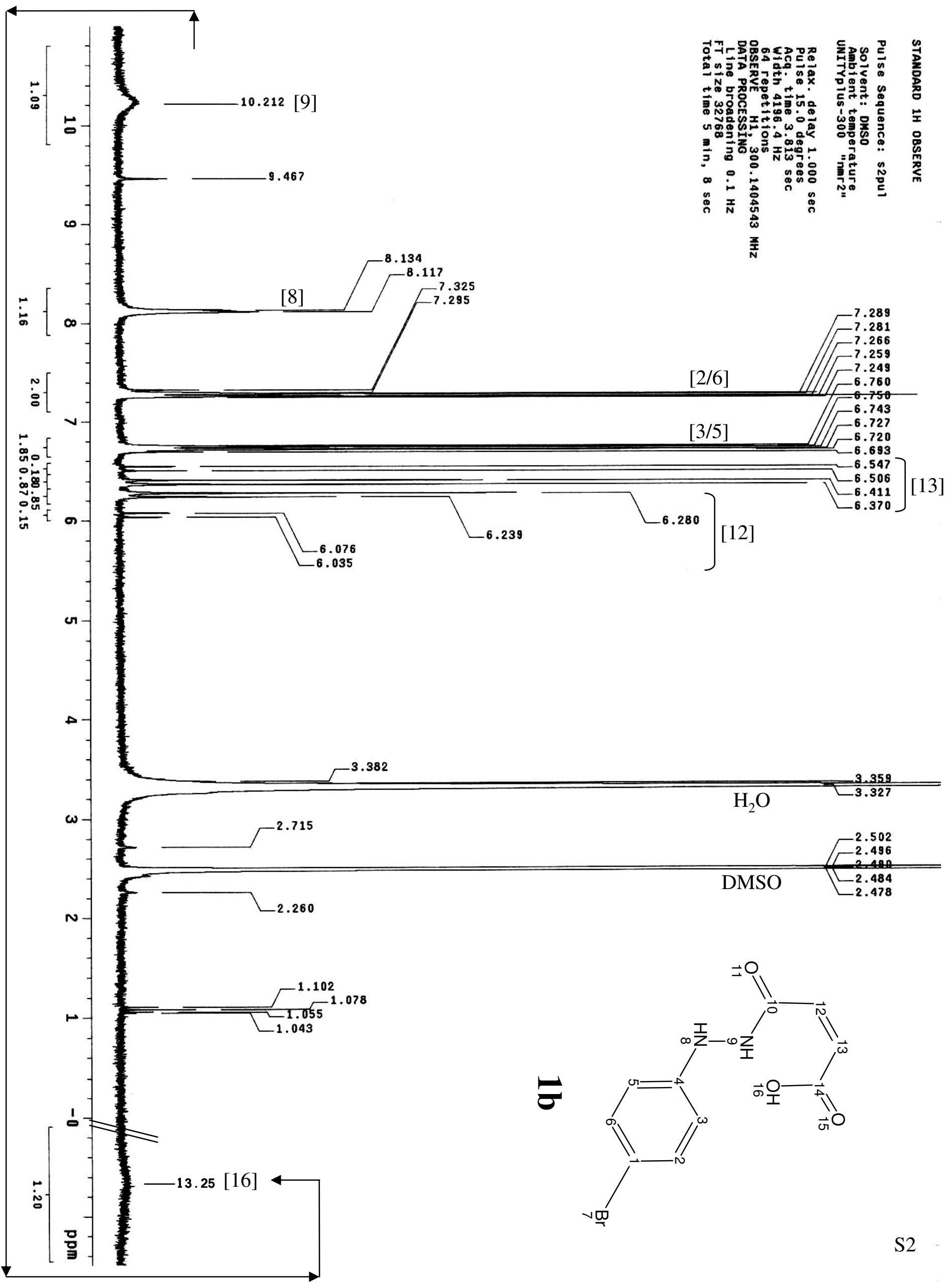


Pulse Sequence: s2pu
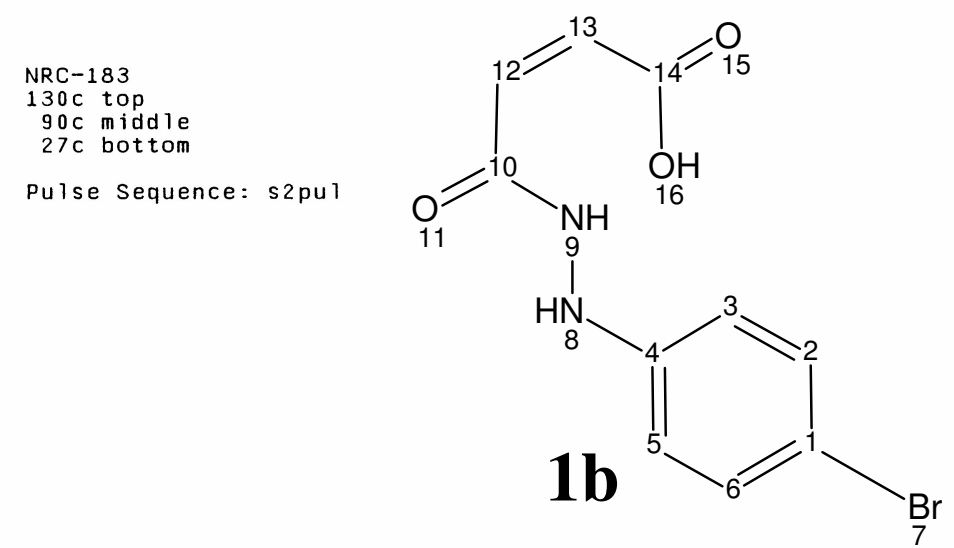

$130{ }^{\circ} \mathrm{C}$

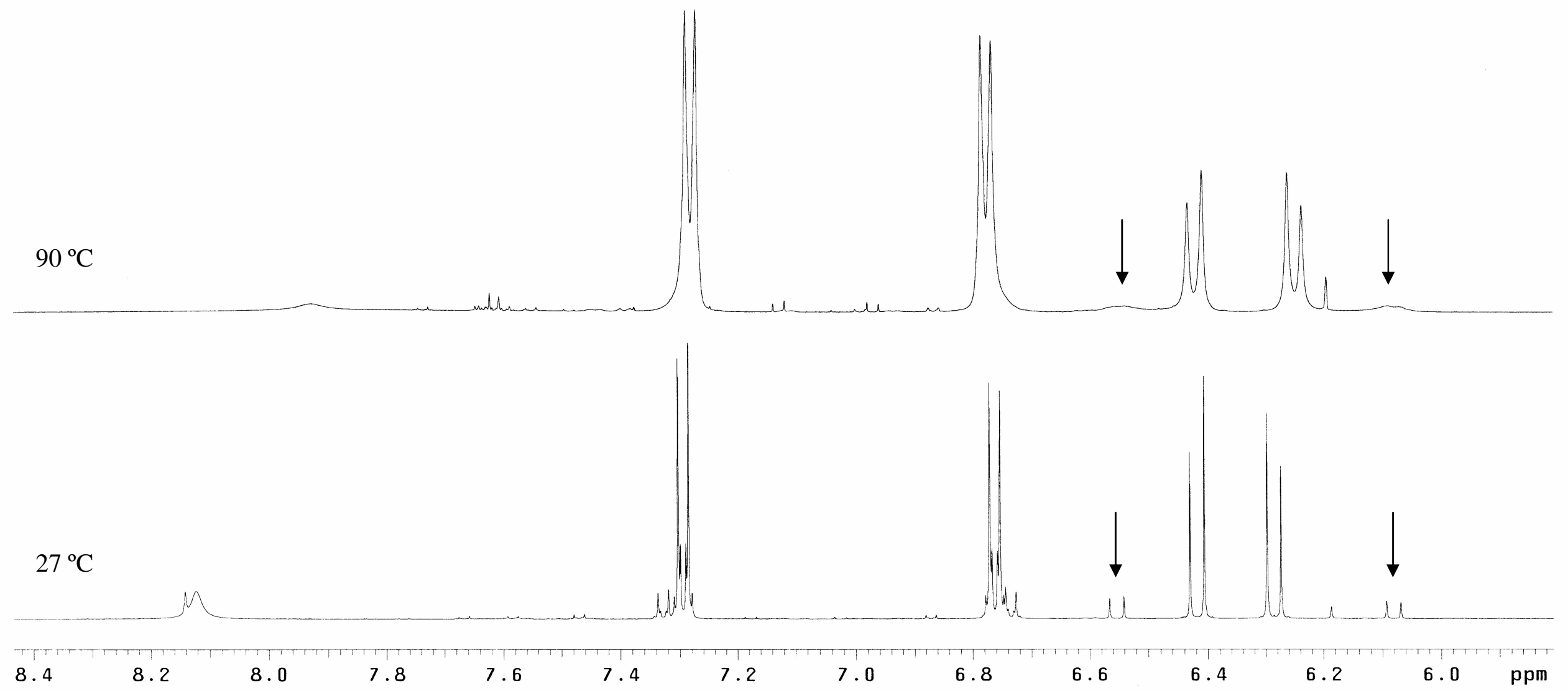


Each olefinic proton appears at two different chemical shifts due to double bond character of "amide" nitrogen.

NRC-183

Pulse Sequence: goesy

NOE/exchange ${ }^{1} \mathrm{H}$ NMR spectrum

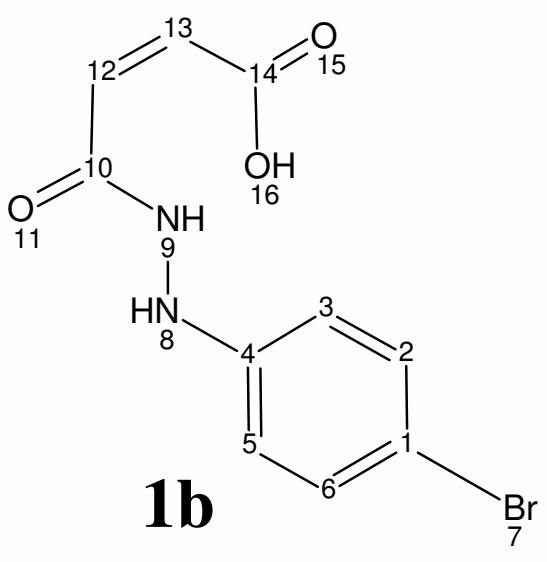

shift enhancement due to NOE

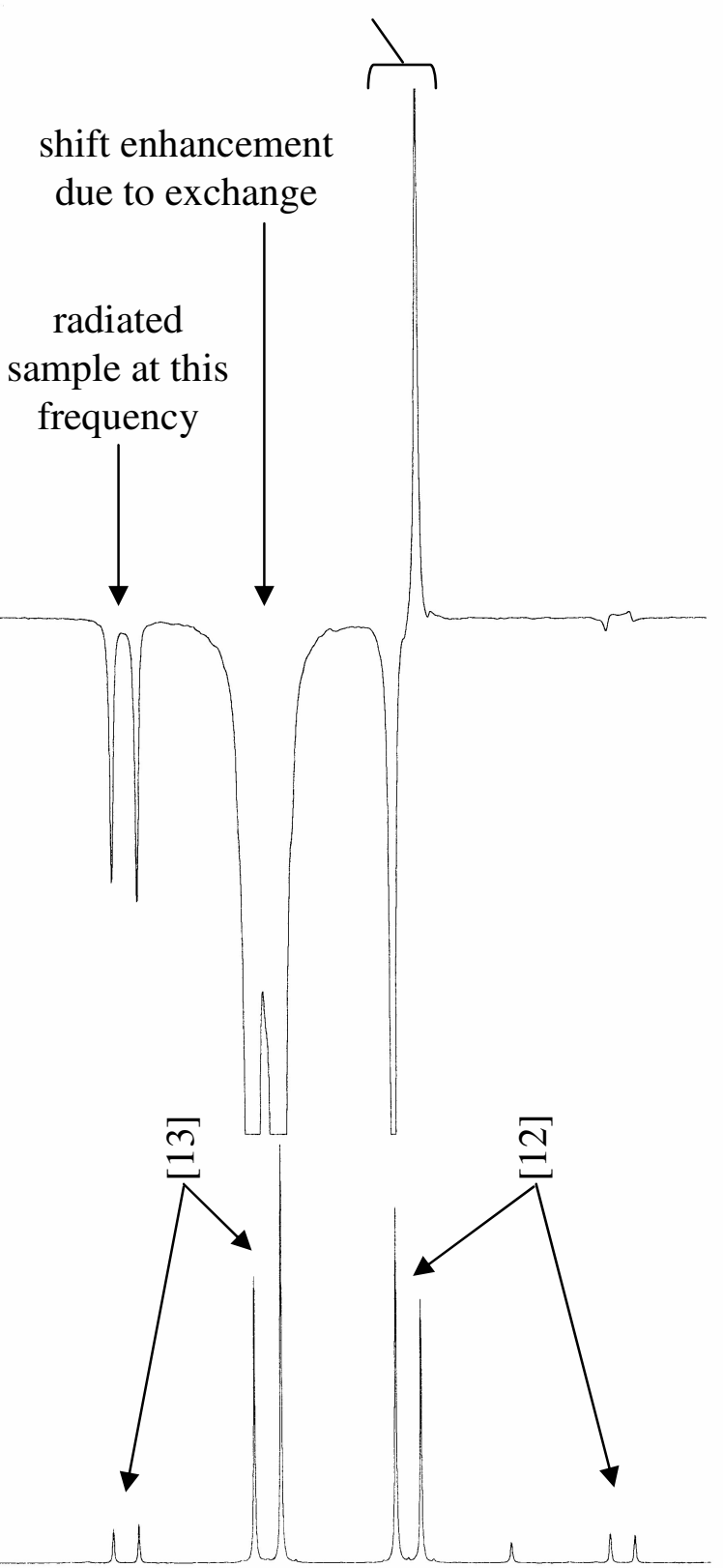

${ }^{1} \mathrm{H}$ NMR spectrum

8.0

7.8

7. 6

7.4

7.2

7.0

6. 8

6.6

6.4

6.2 
Pulse Sequence: s2pul

Solvent: DMSO

Amblent temperature
UNITYp Ius-300 "nmr3"

Relax. delay $1.000 \mathrm{sec}$

Acq. $t$ ime $1.814 \mathrm{sec}$

Width $20000.0 \mathrm{~Hz}$

344 repetitions

OBSERVE C13, 75.4072514 MHZ

DECOUPLE H1, 299.8920521 MHZ

Power $40 \mathrm{~dB}$
cont inuous $1 \mathrm{y}$

cont inuous Iy on

single precision data

DATA PROCESSING

line broadening $3.0 \mathrm{~Hz}$

Total time $1 \mathrm{hr}, 16 \mathrm{sec}$

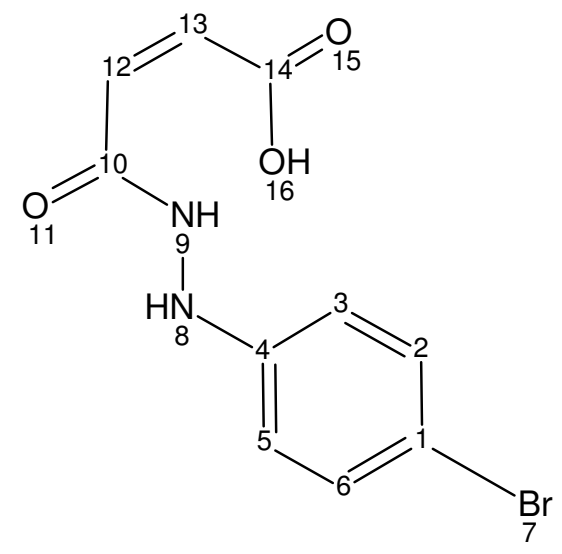

$1 b$

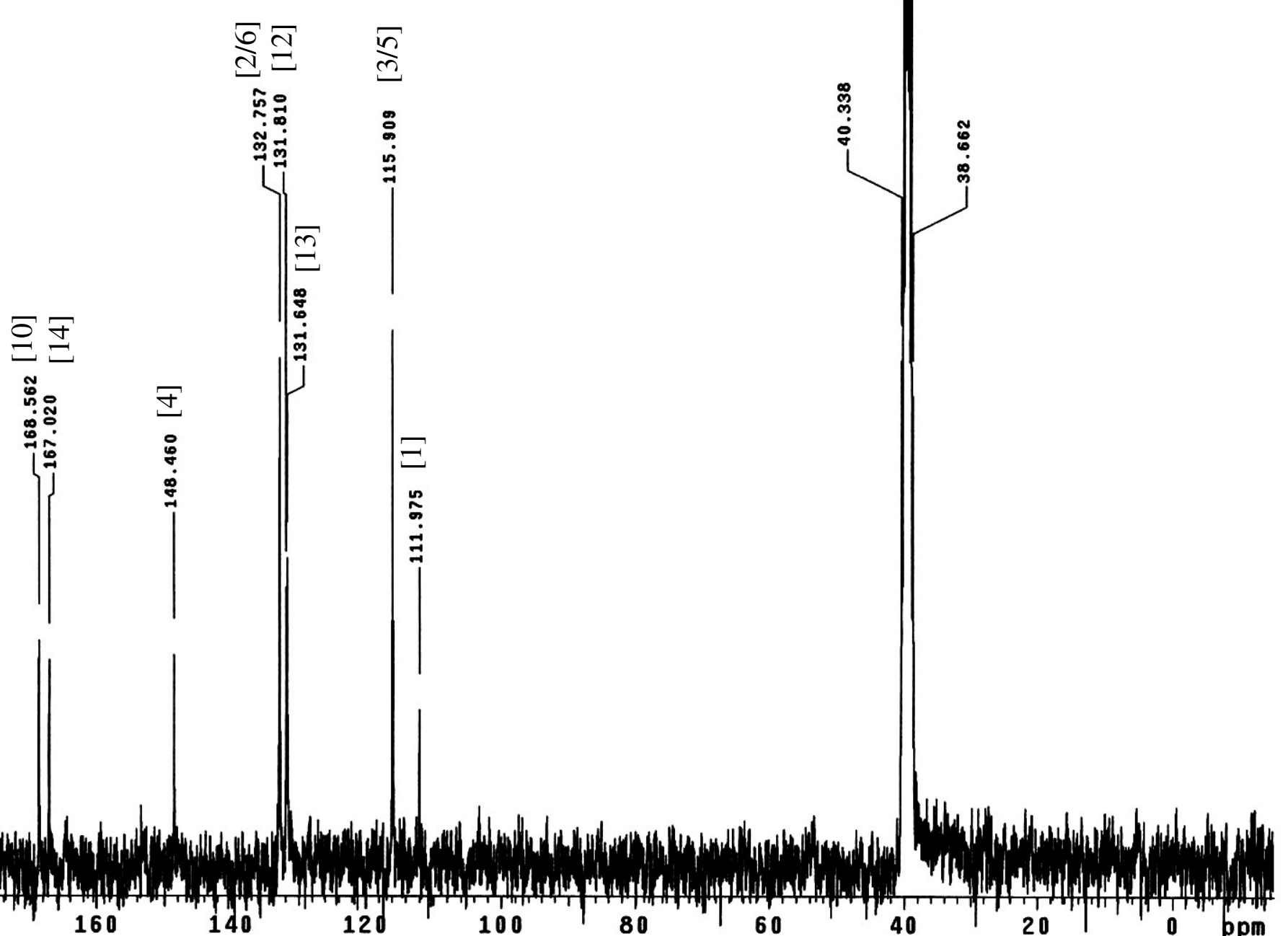

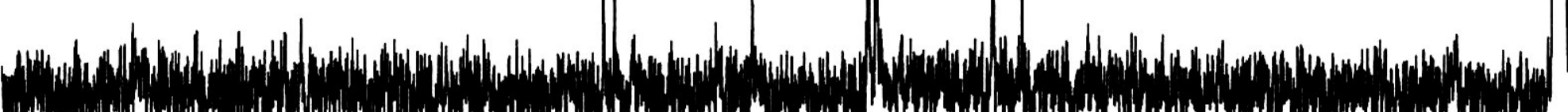

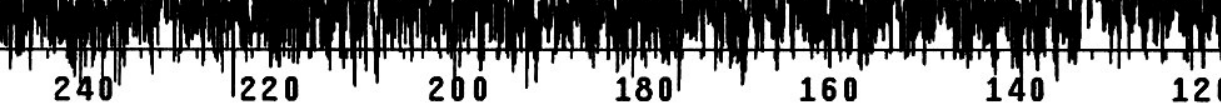


IR (KBr) spectrum of $\mathbf{1 b}$

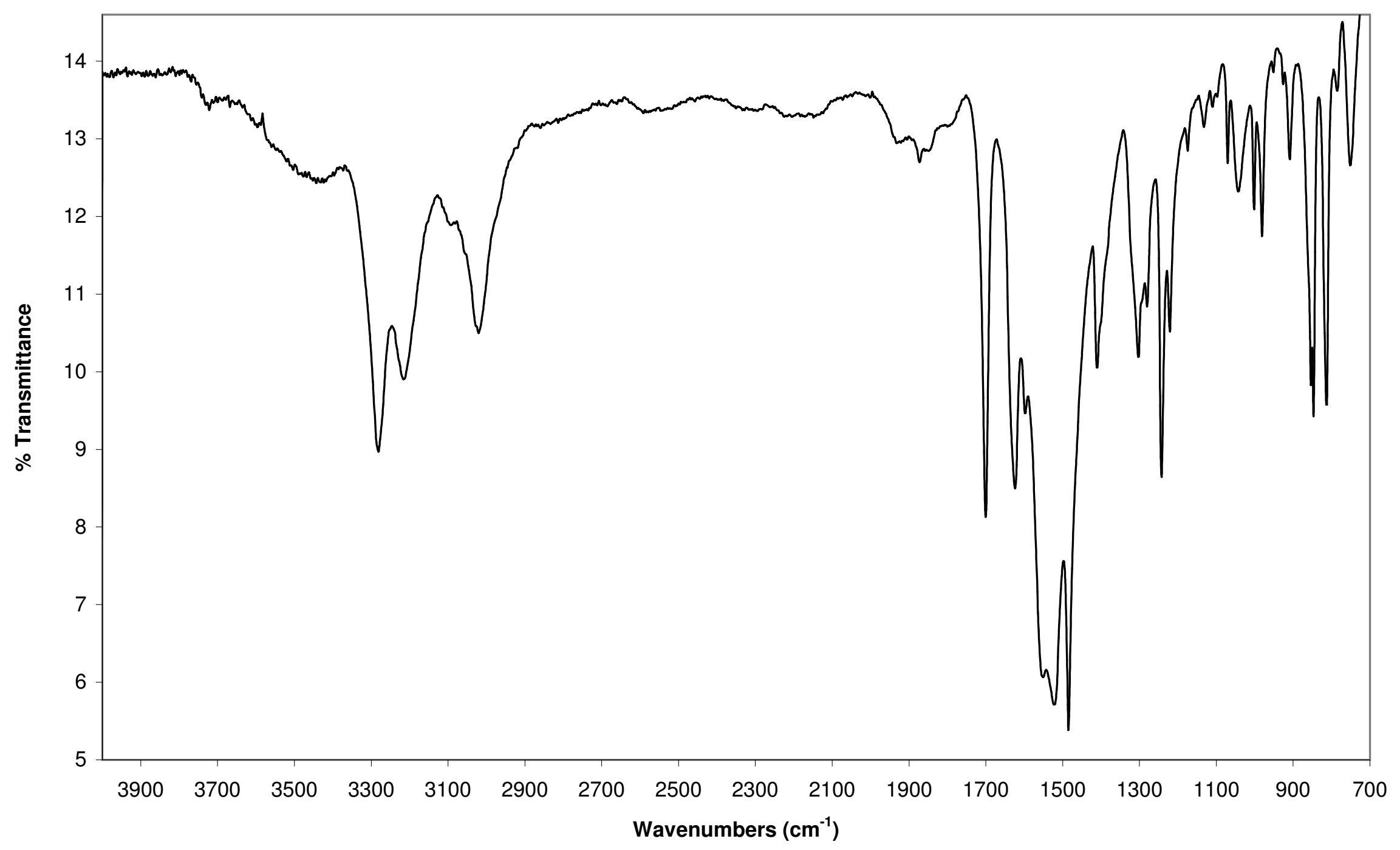


NRC-17S

Pulse Sequence: s2pul

Solvent: DMSO

remp. $27.0 \mathrm{C} / 300.1 \mathrm{~K}$

File:-nrc_17s_h1

Relax. delay $2.000 \mathrm{sec}$ Acq. time $4.865 \mathrm{sec}$

Width $6577.3 \mathrm{~Hz}$

32 repetitions

OBSERVE H1, $499.3490042 \mathrm{MHZ}$

(ne

作

Total time $4 \mathrm{~min}, 7 \mathrm{sec}$

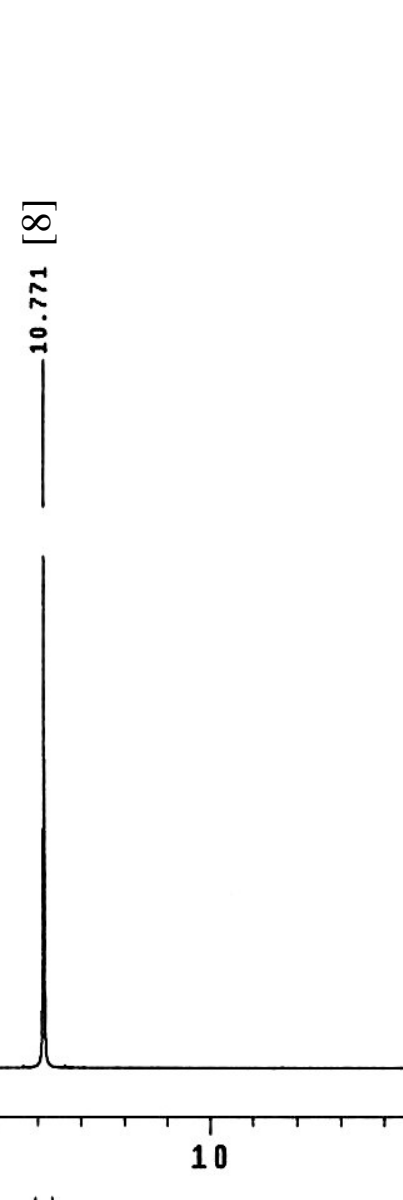

1.00

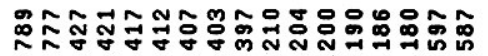
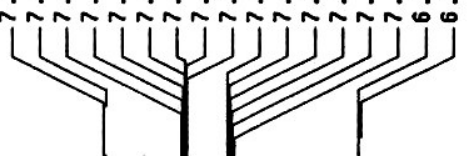

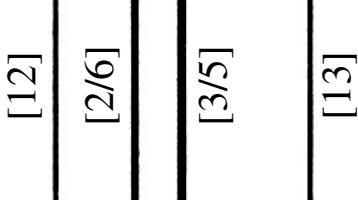

(1)

8

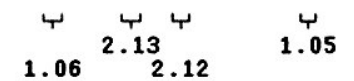

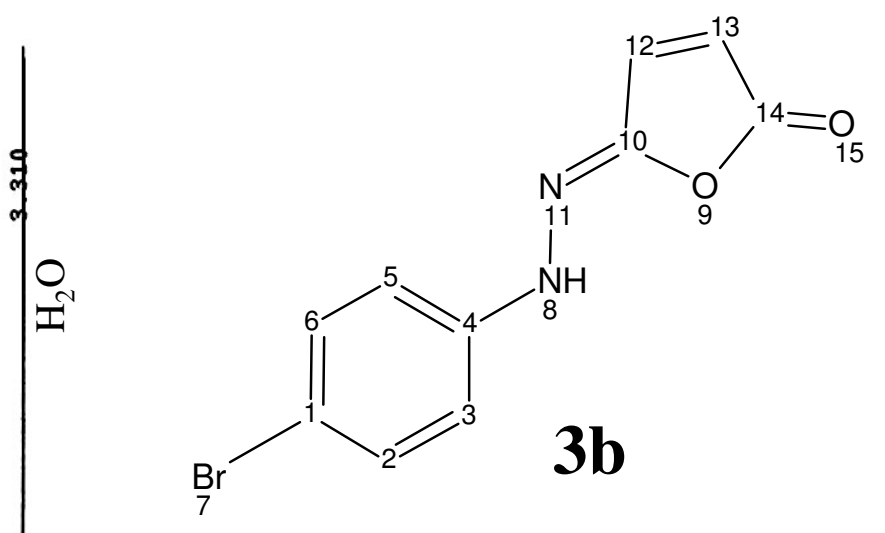

ลั่ 
NRC-17S

Pulse Sequence: gHSOC

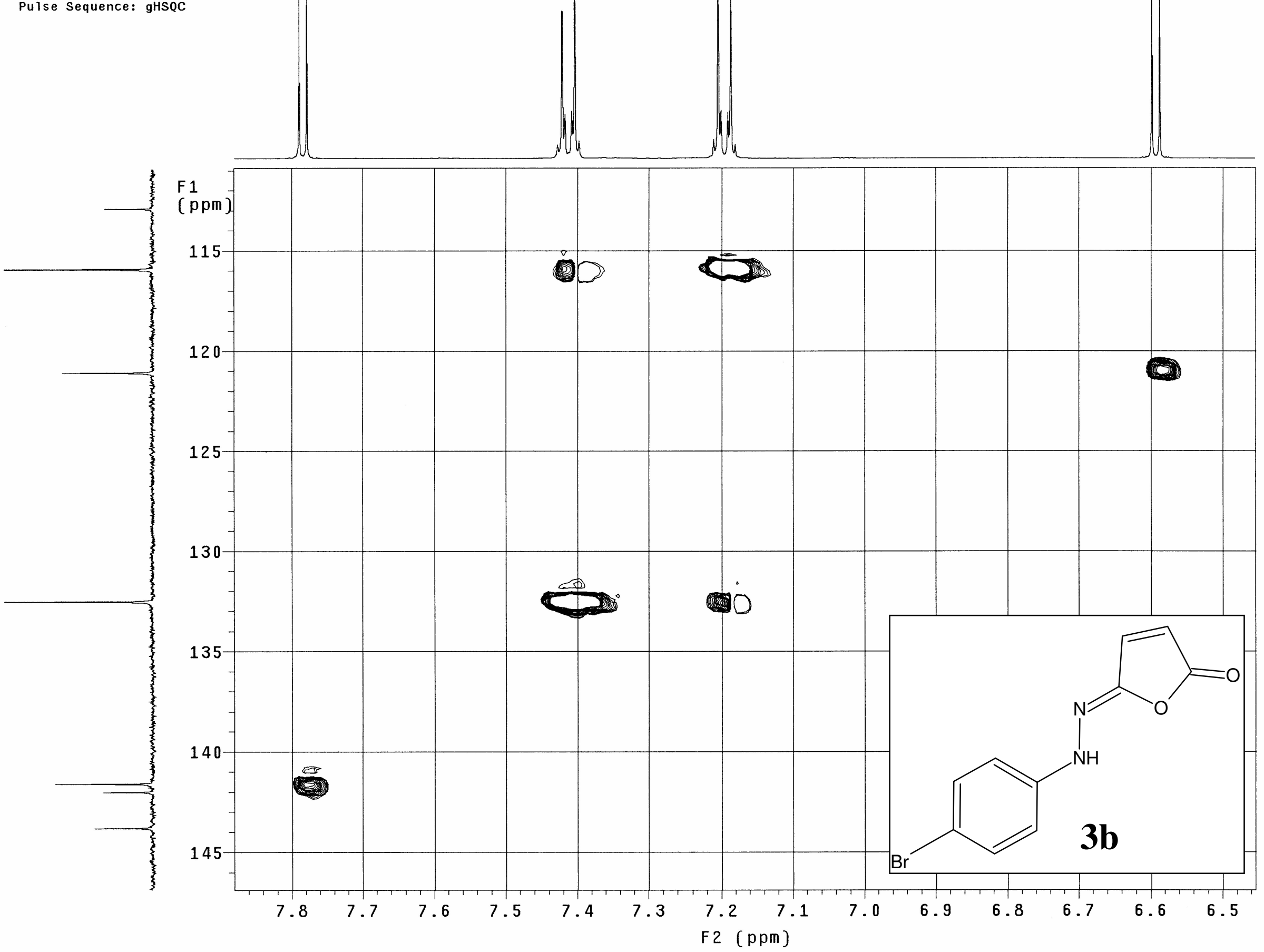




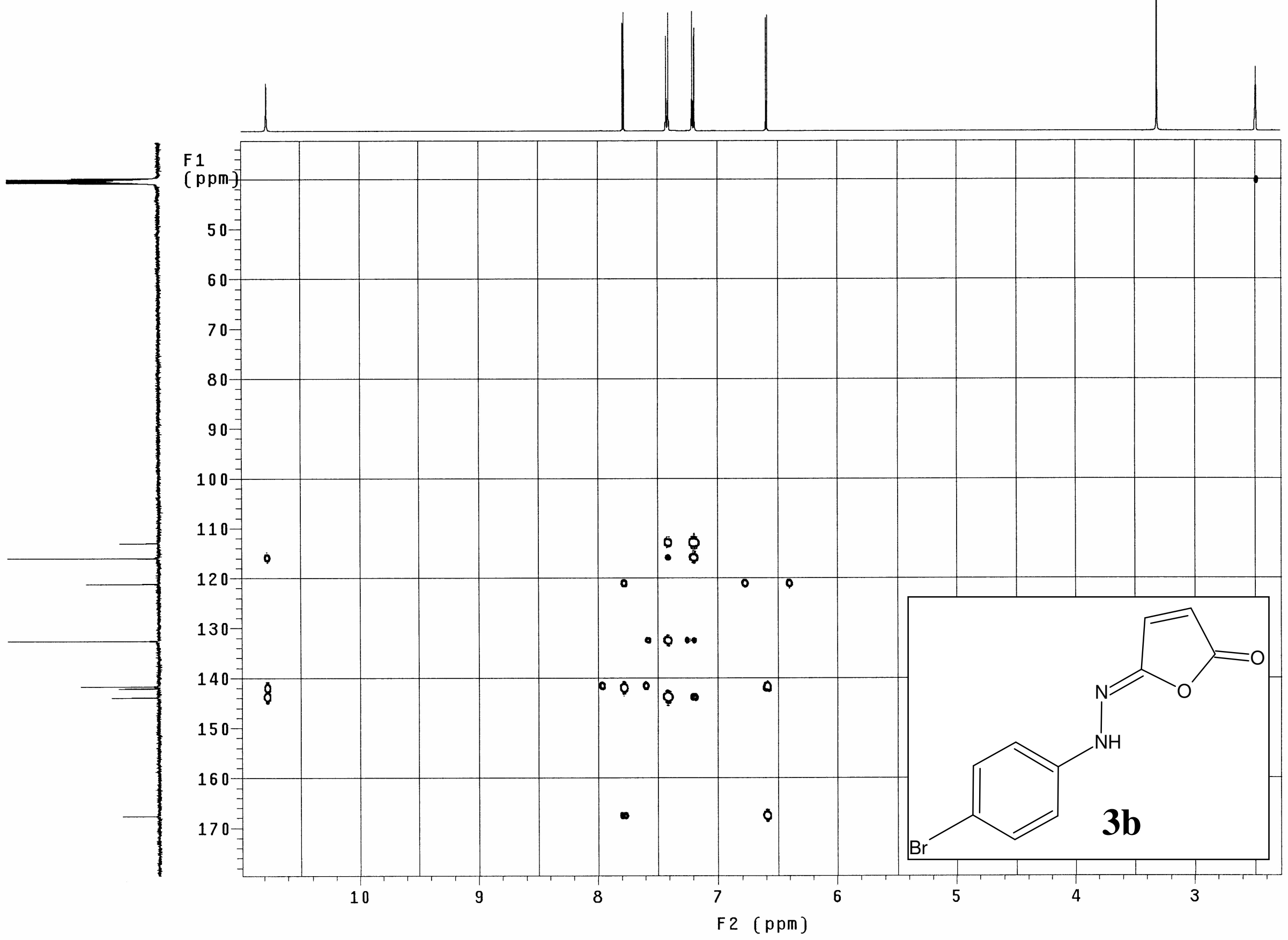


NRC-17S

Pulse Sequence: s2pul solvent is DMSO- $\mathrm{d}_{6}$

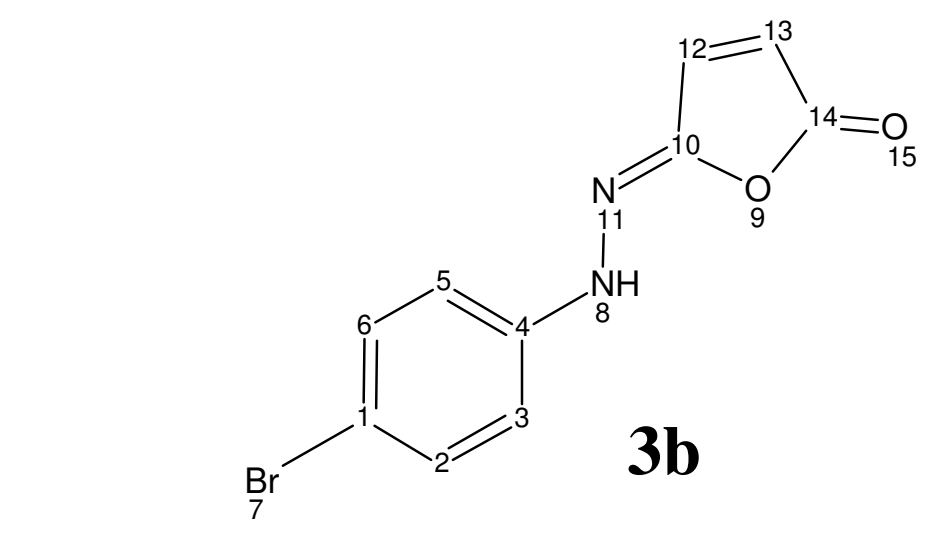

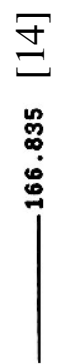
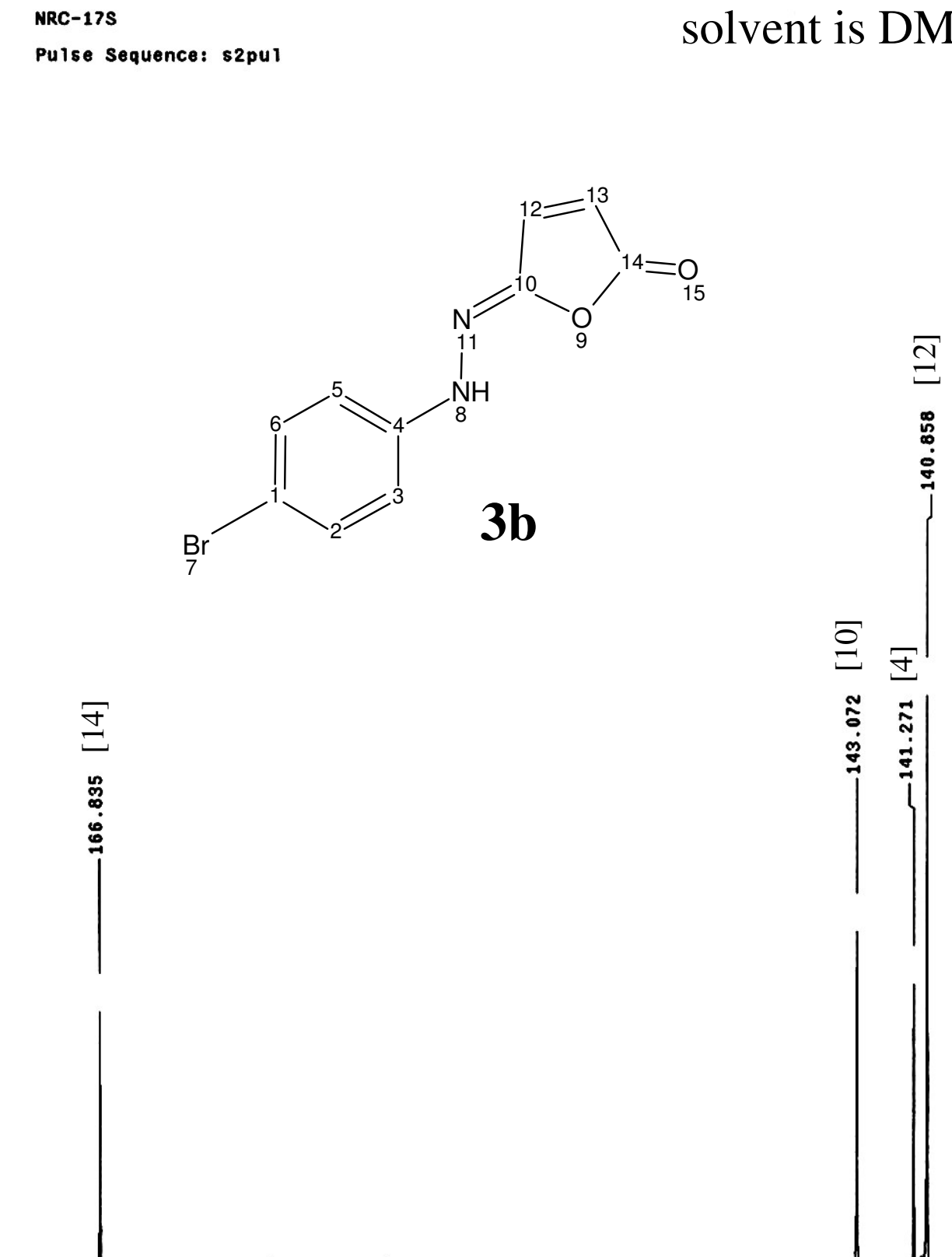

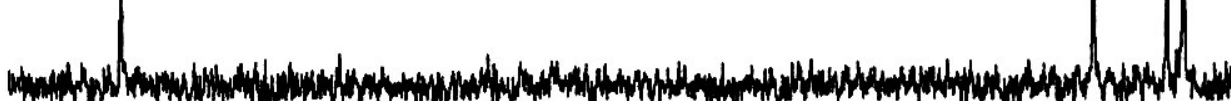

(n)

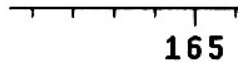


IR $(\mathrm{KBr})$ spectrum of compound $\mathbf{3 b}$

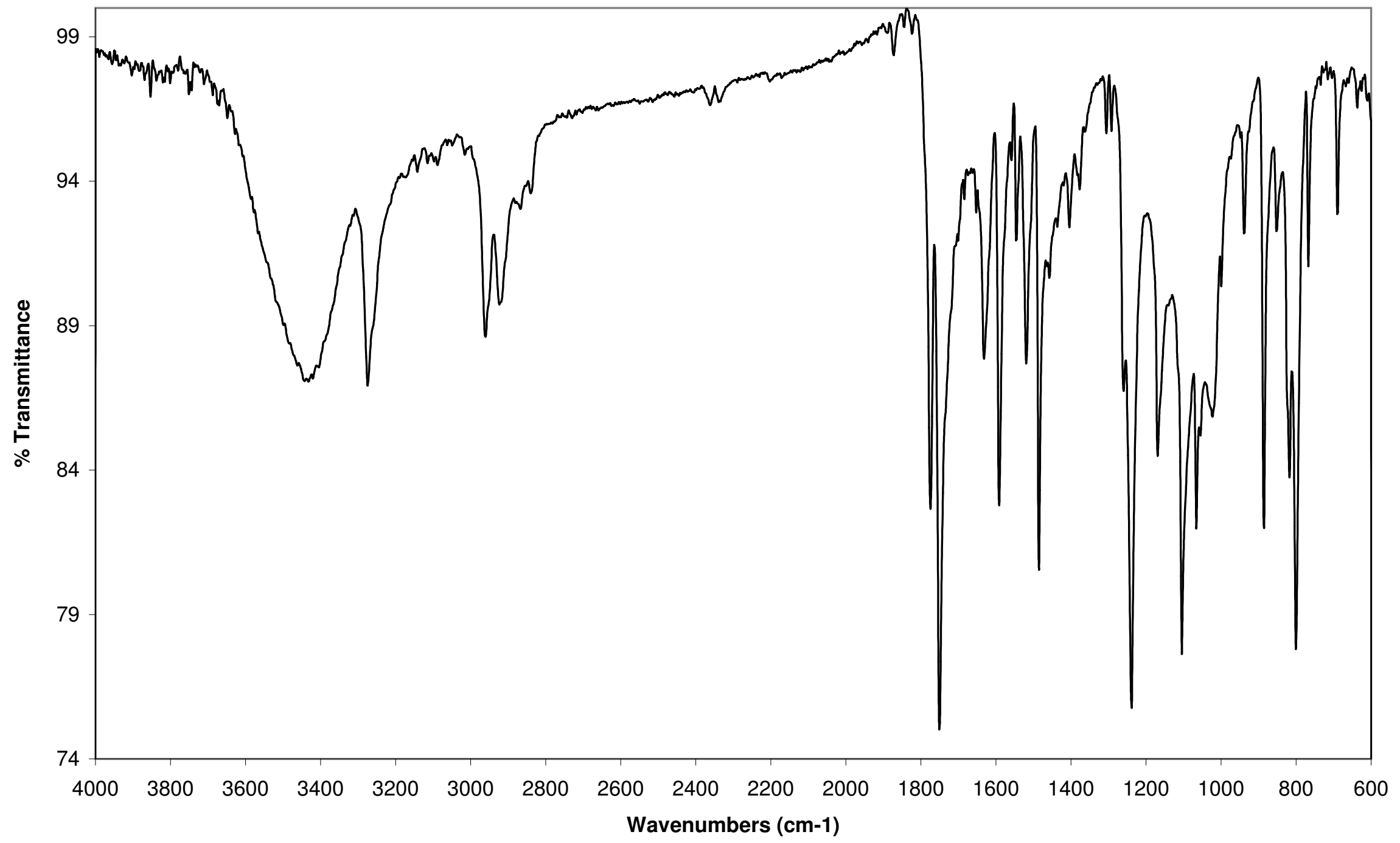




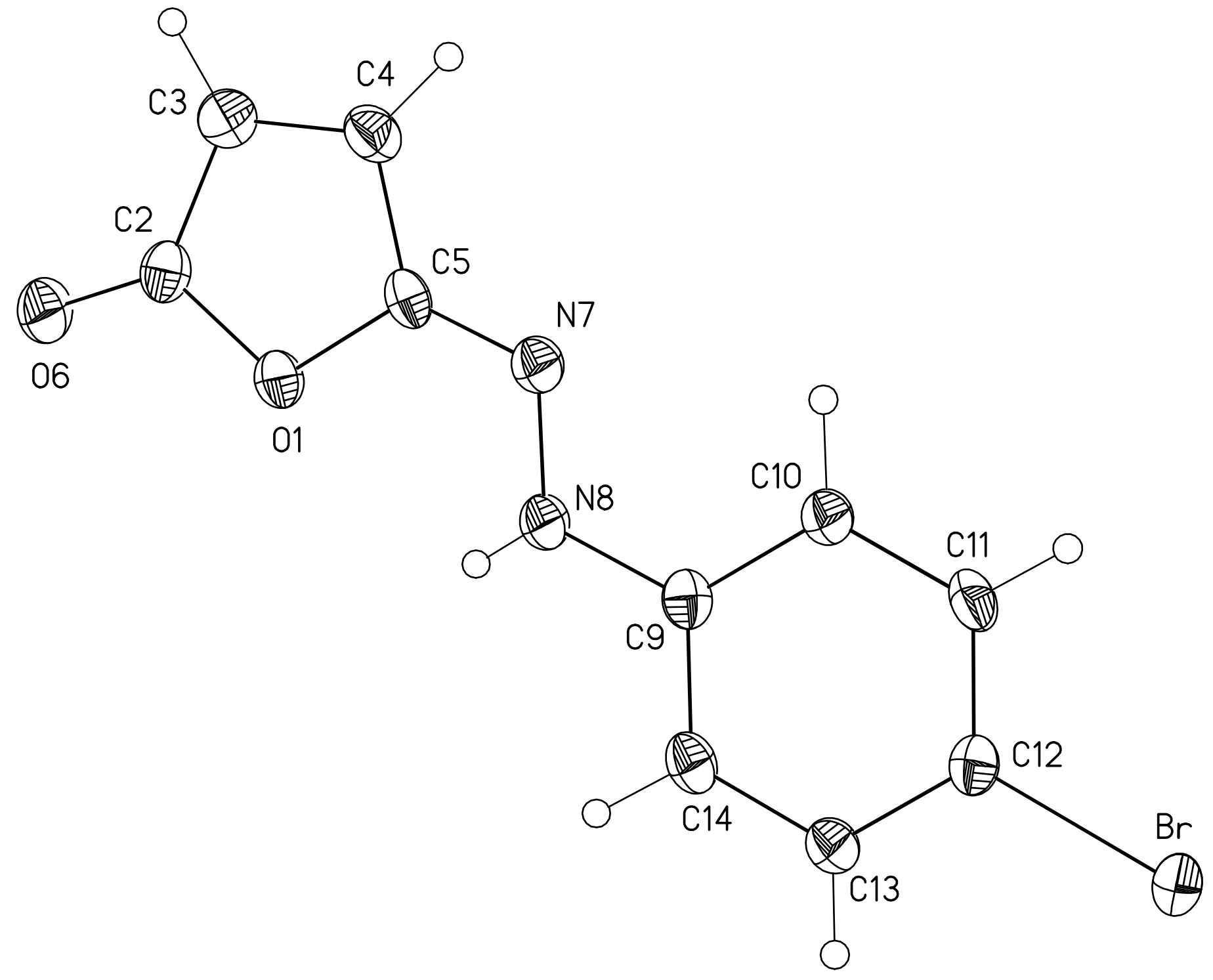

Molecular structure of $\mathbf{3 b}$ showing the atomic labeling scheme with thermal ellipsoids (50\% probability). 


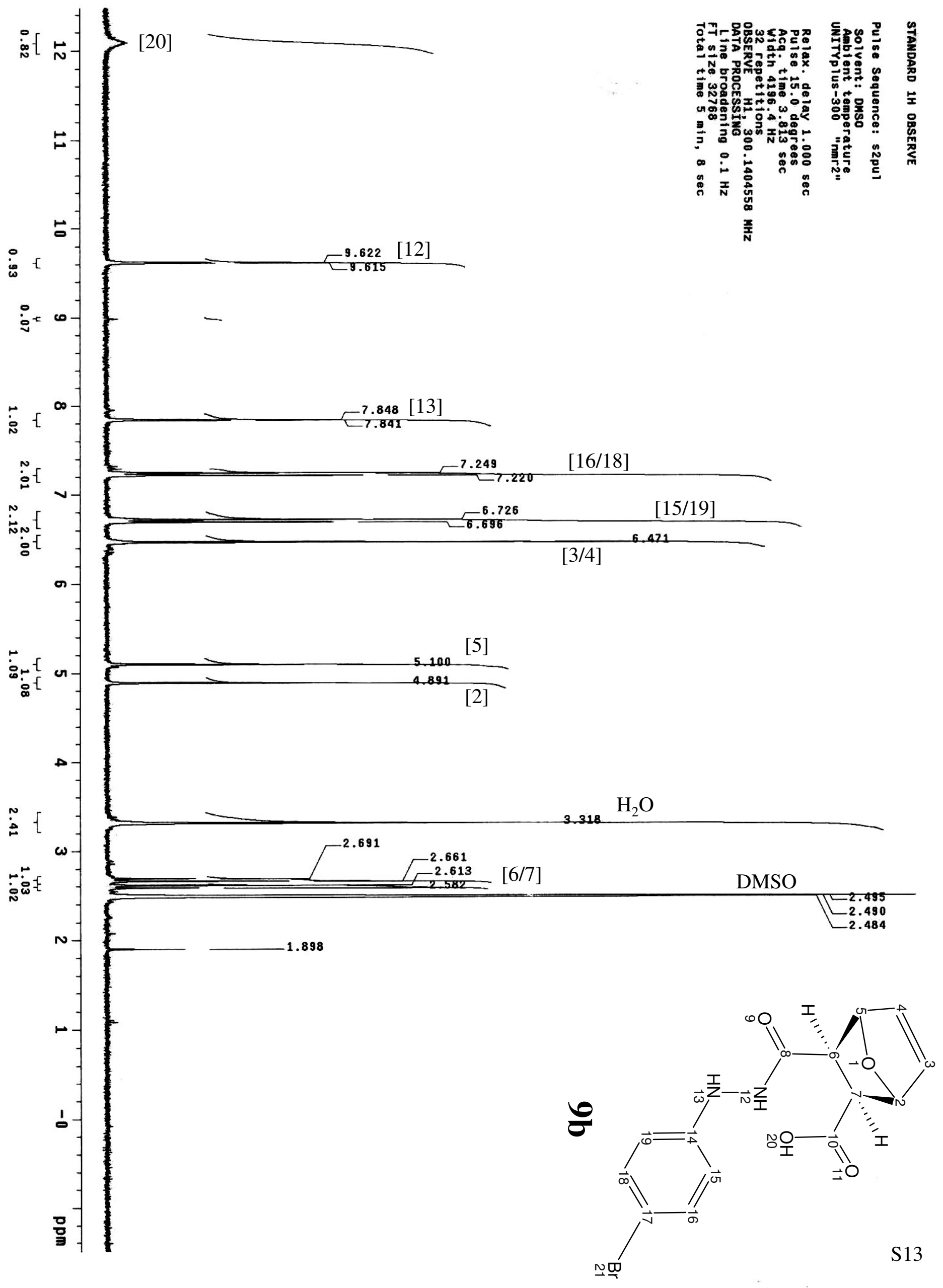




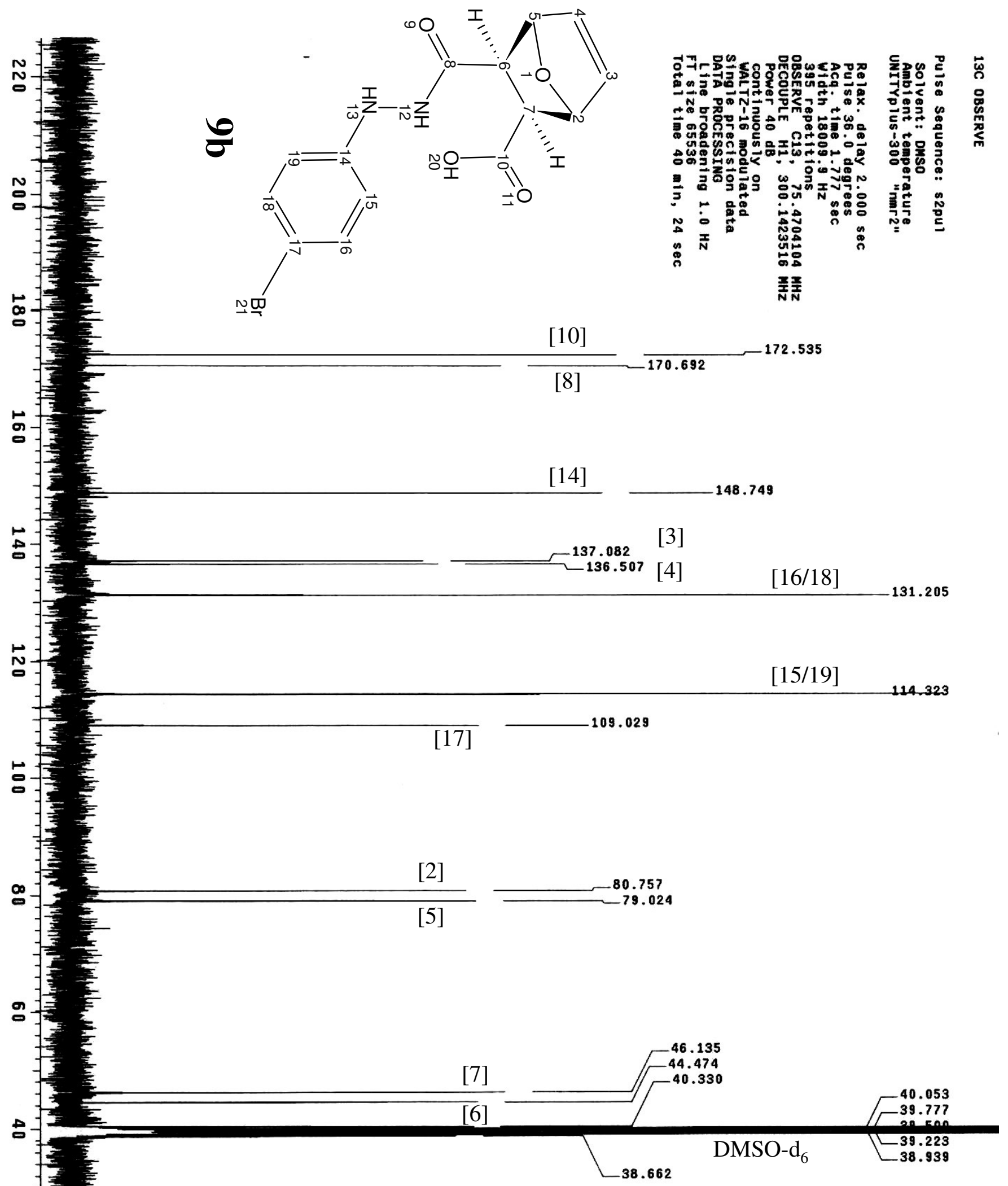


IR $(\mathrm{KBr})$ spectrum of compound $\mathbf{9 b}$

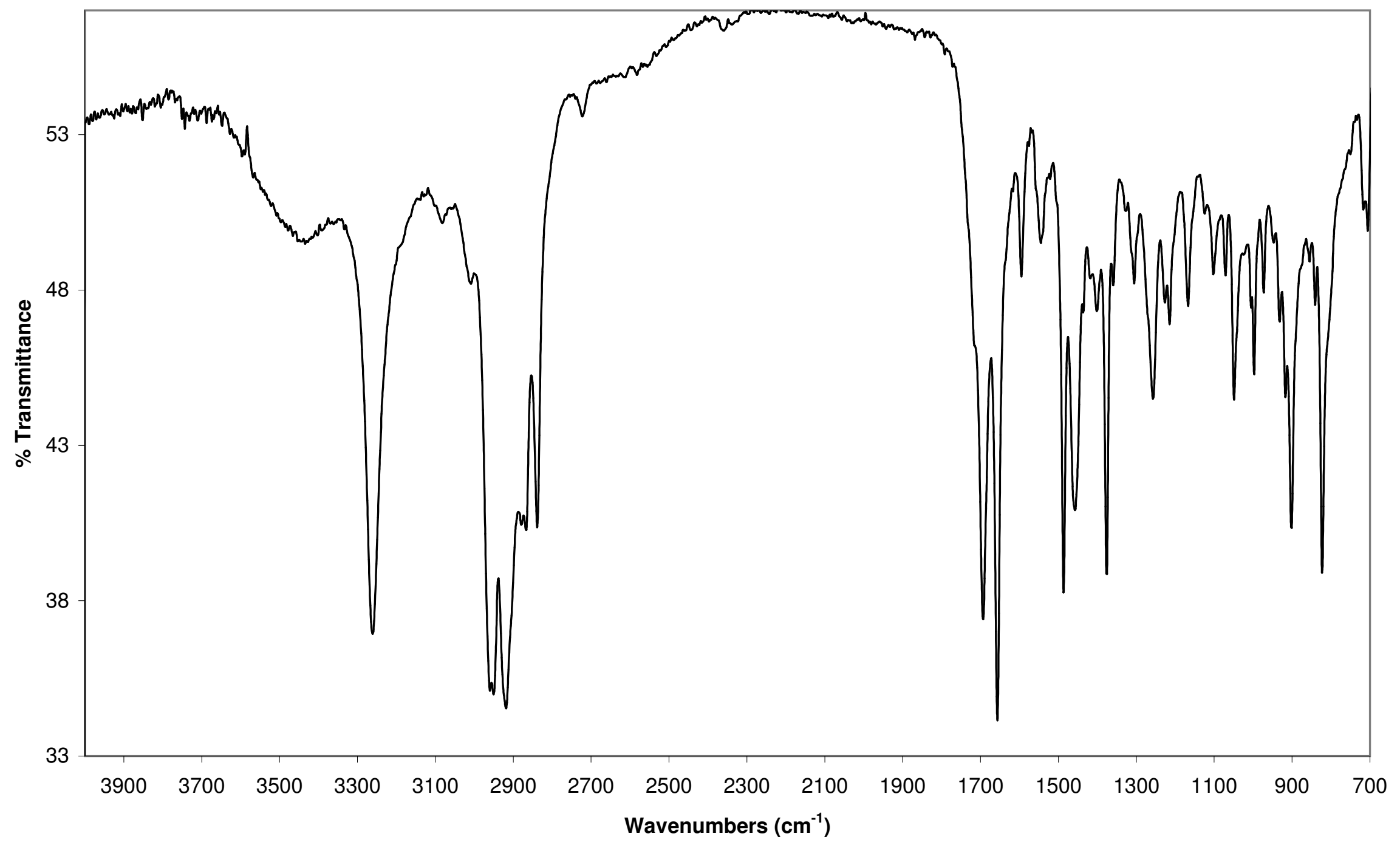




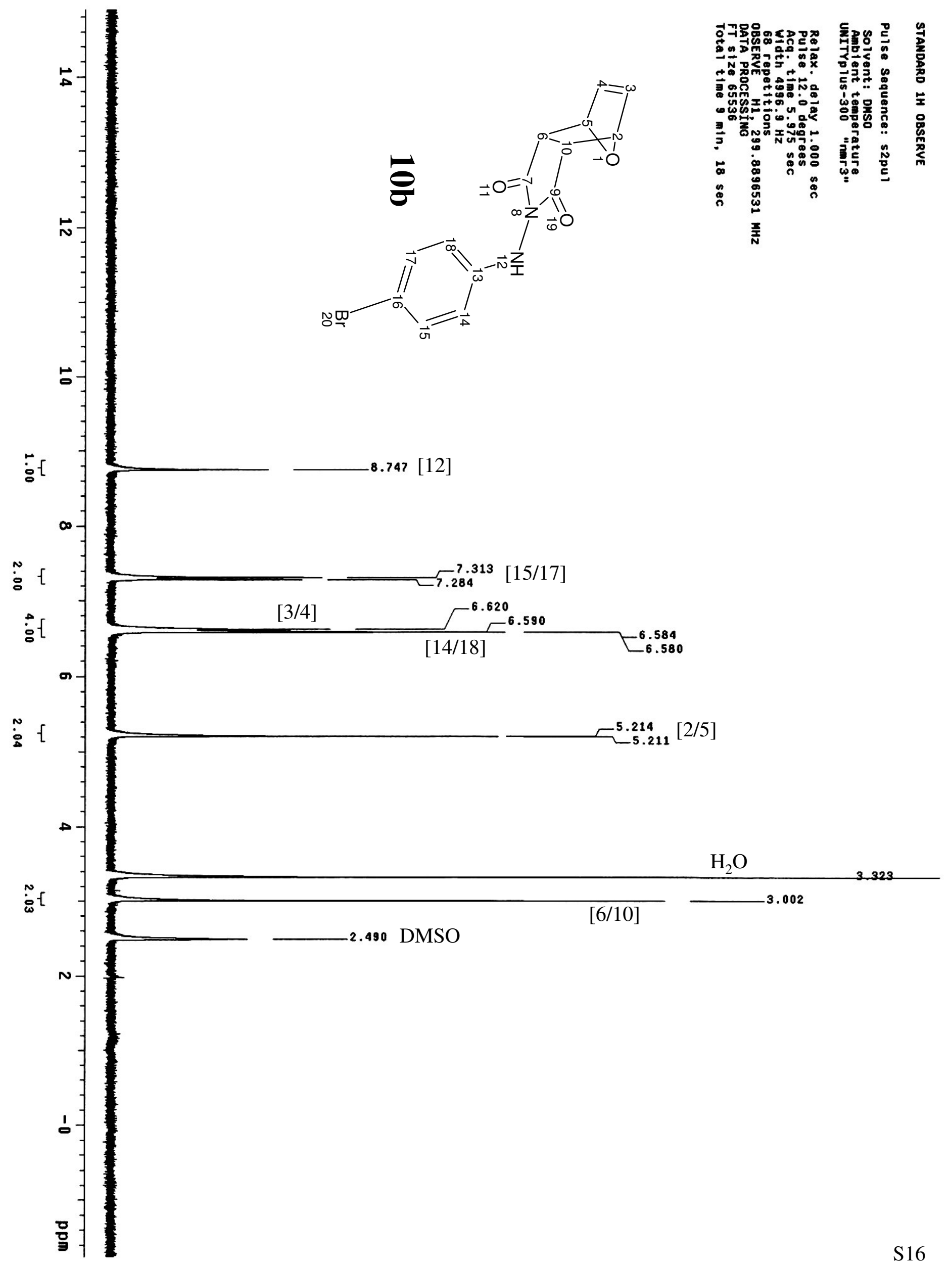




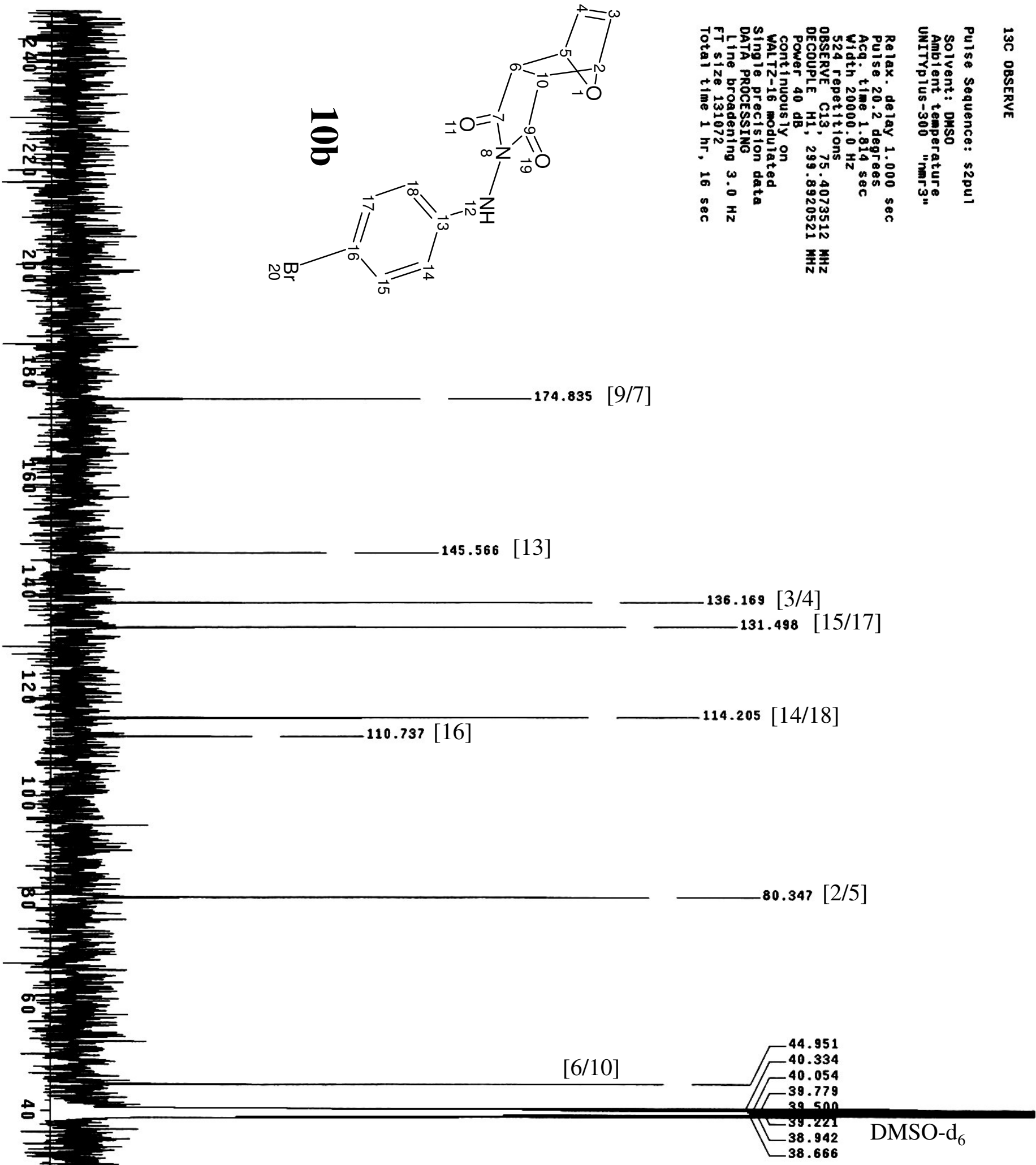




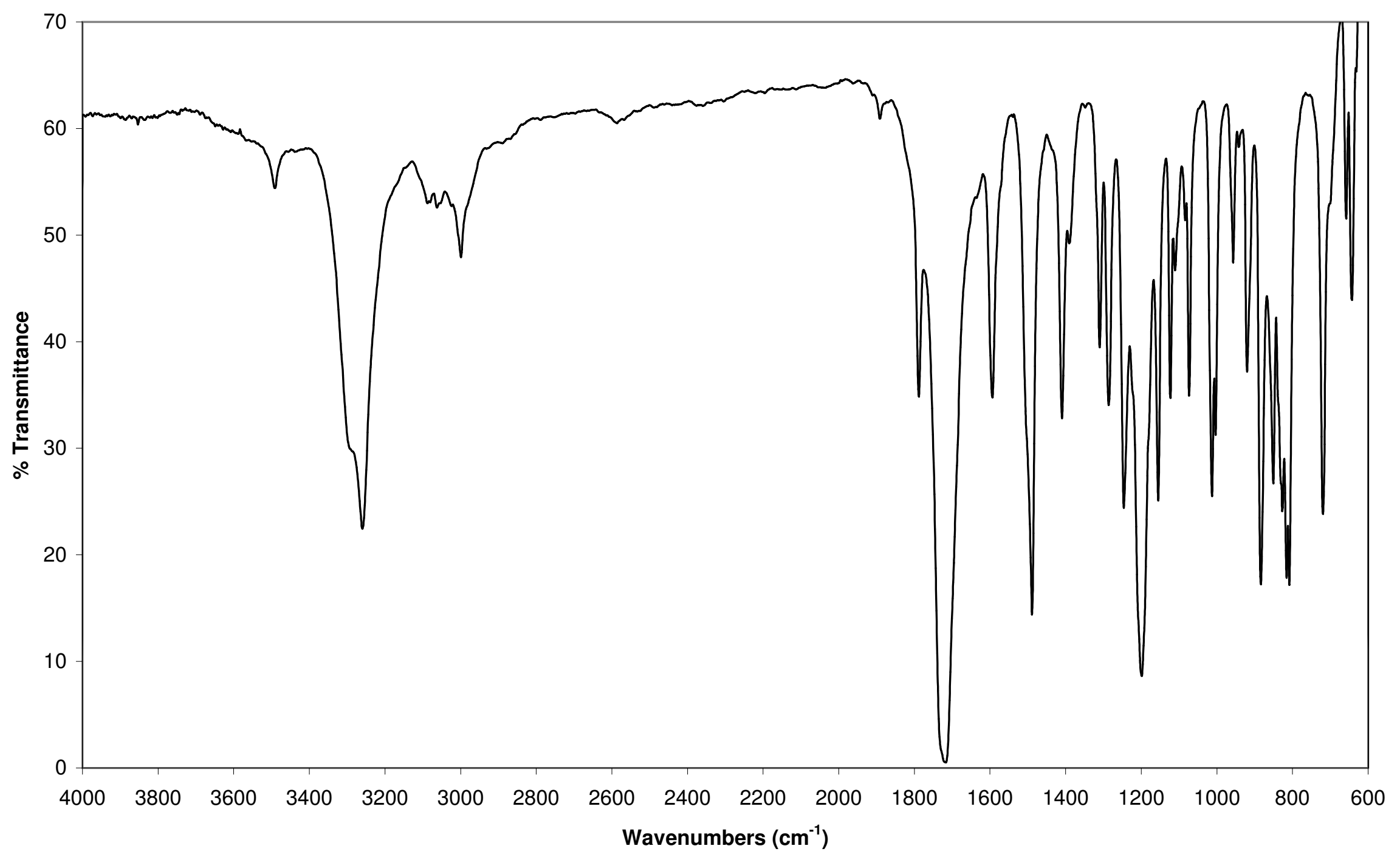




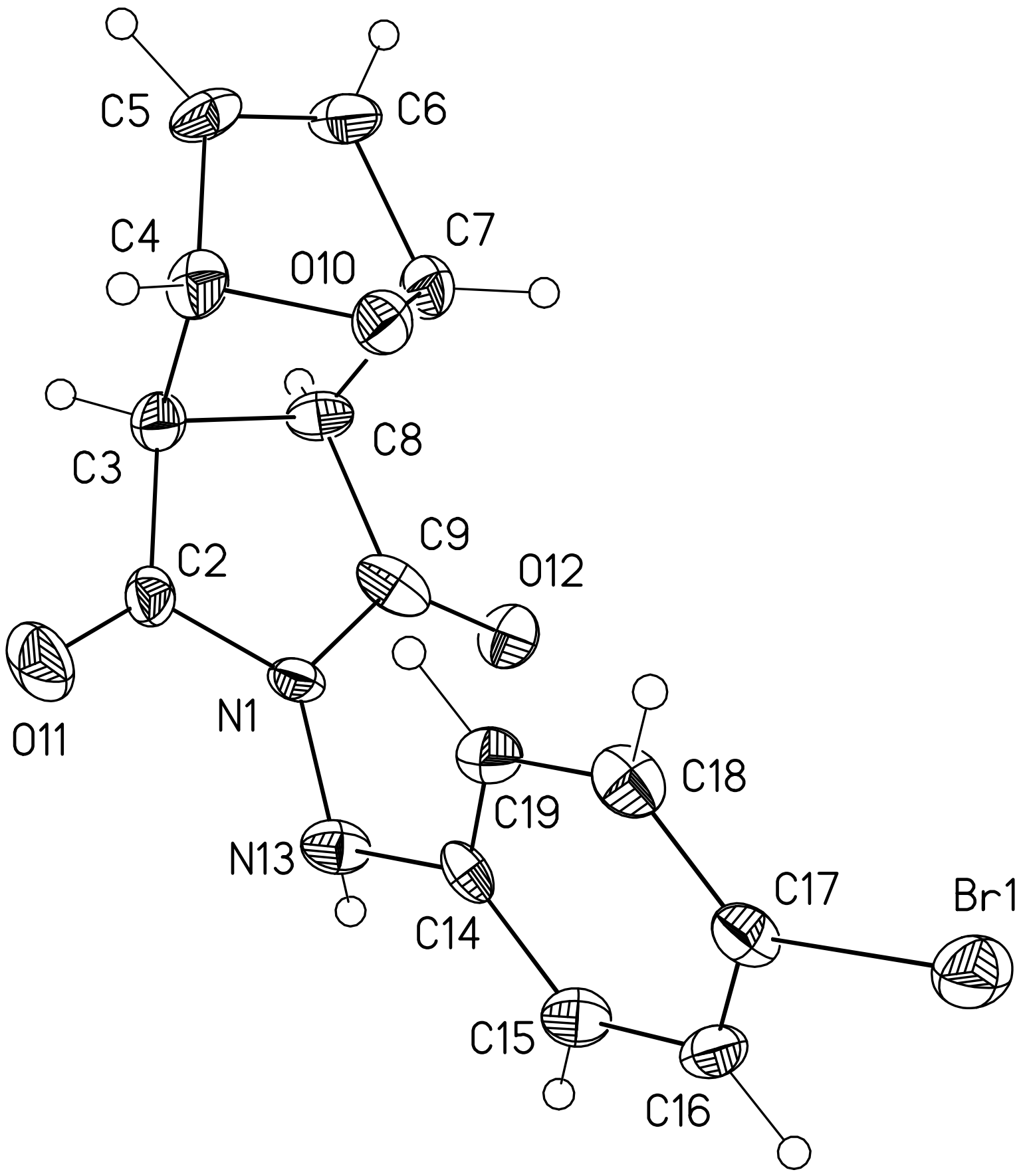

Molecular structure of $\mathbf{1 0 b}$ showing the atomic labeling scheme with thermal ellipsoids (50\% probability). 
STANDARD IH OBSERVE

Pulse Sequence: s2pul

Solvent: DMSO

Amblent temperature

Re lax. de lay $1.000 \mathrm{sec}$

Pulse 12.0 degrees

Width $4996.9 \mathrm{~Hz}$

OBSERVE H1 299.8896519 MHZ

DATA PROCESSIN

Total time $9 \mathrm{~min}, 18 \mathrm{sec}$ $\underset{⿱ 亠 乂}{\stackrel{8}{2}}$

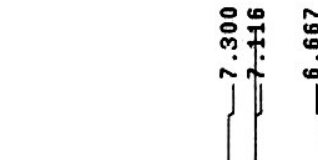

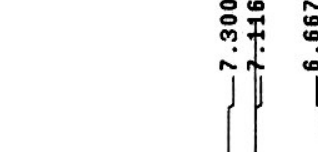

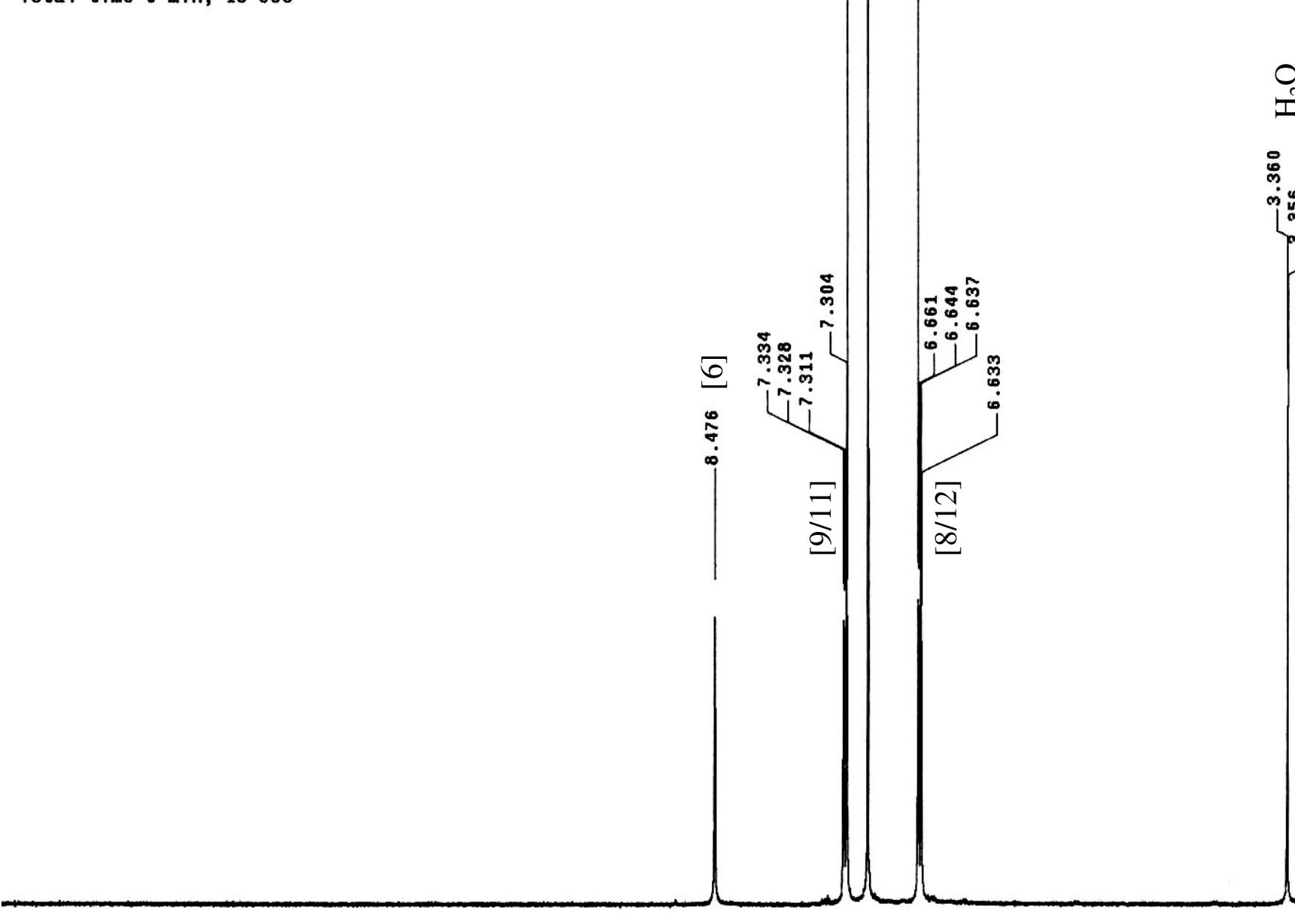

12
10
8

1.00
6 $\begin{array}{cc}2.05 \\ 2.07 & 2.06\end{array}$

$\overbrace{}^{N}$

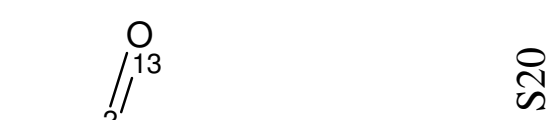

$\sum_{0}^{0}$

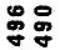

2b

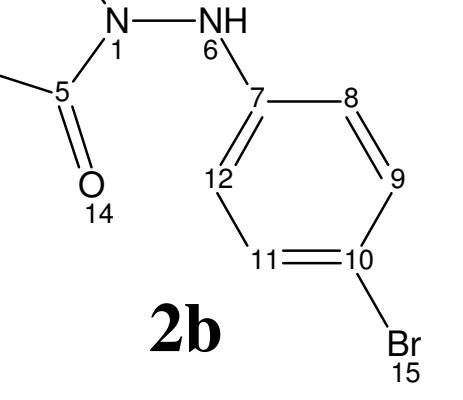

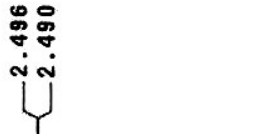

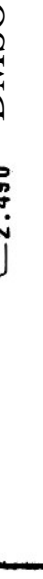




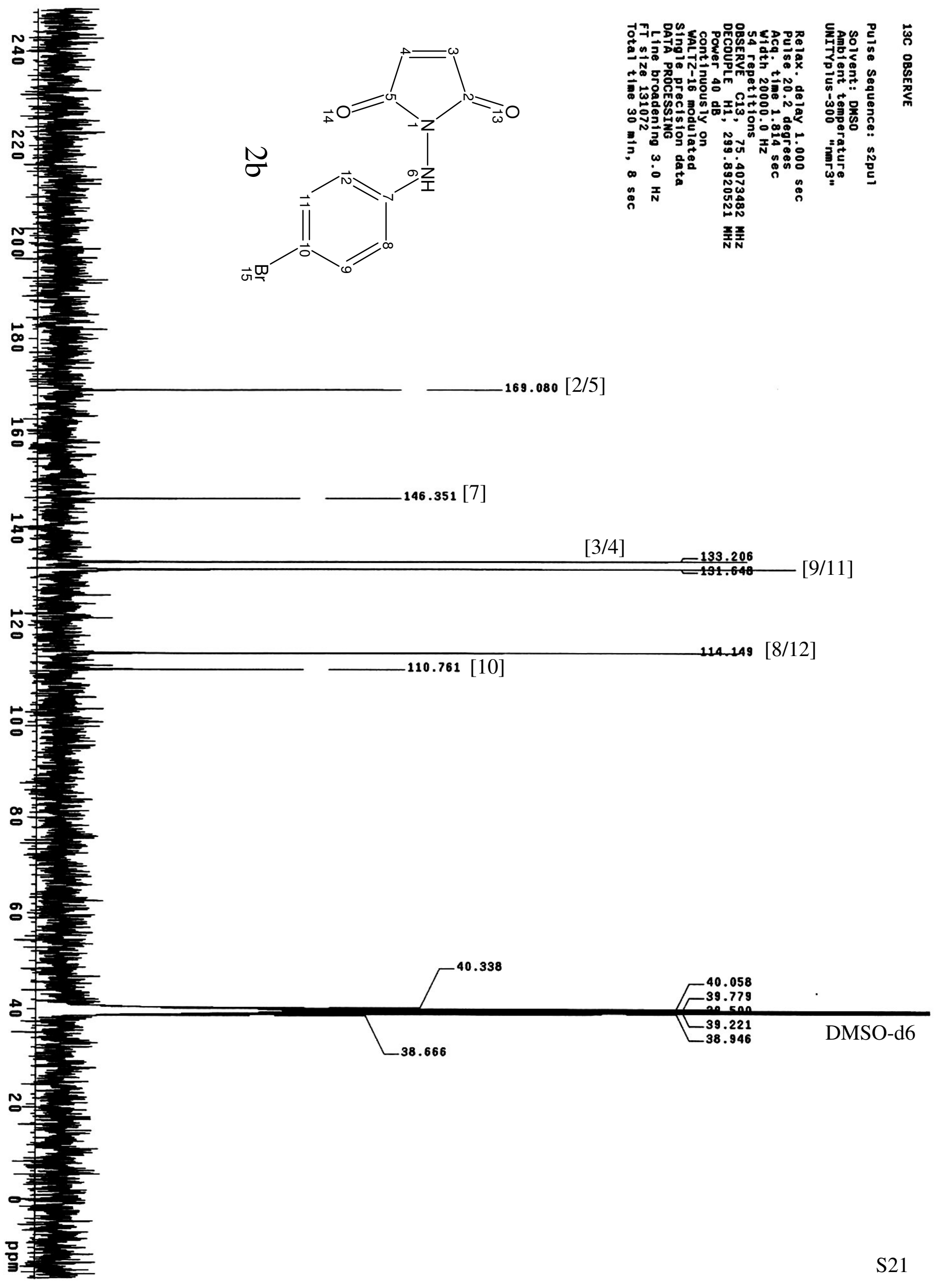


IR (KBr) spectrum of compound $\mathbf{2 b}$

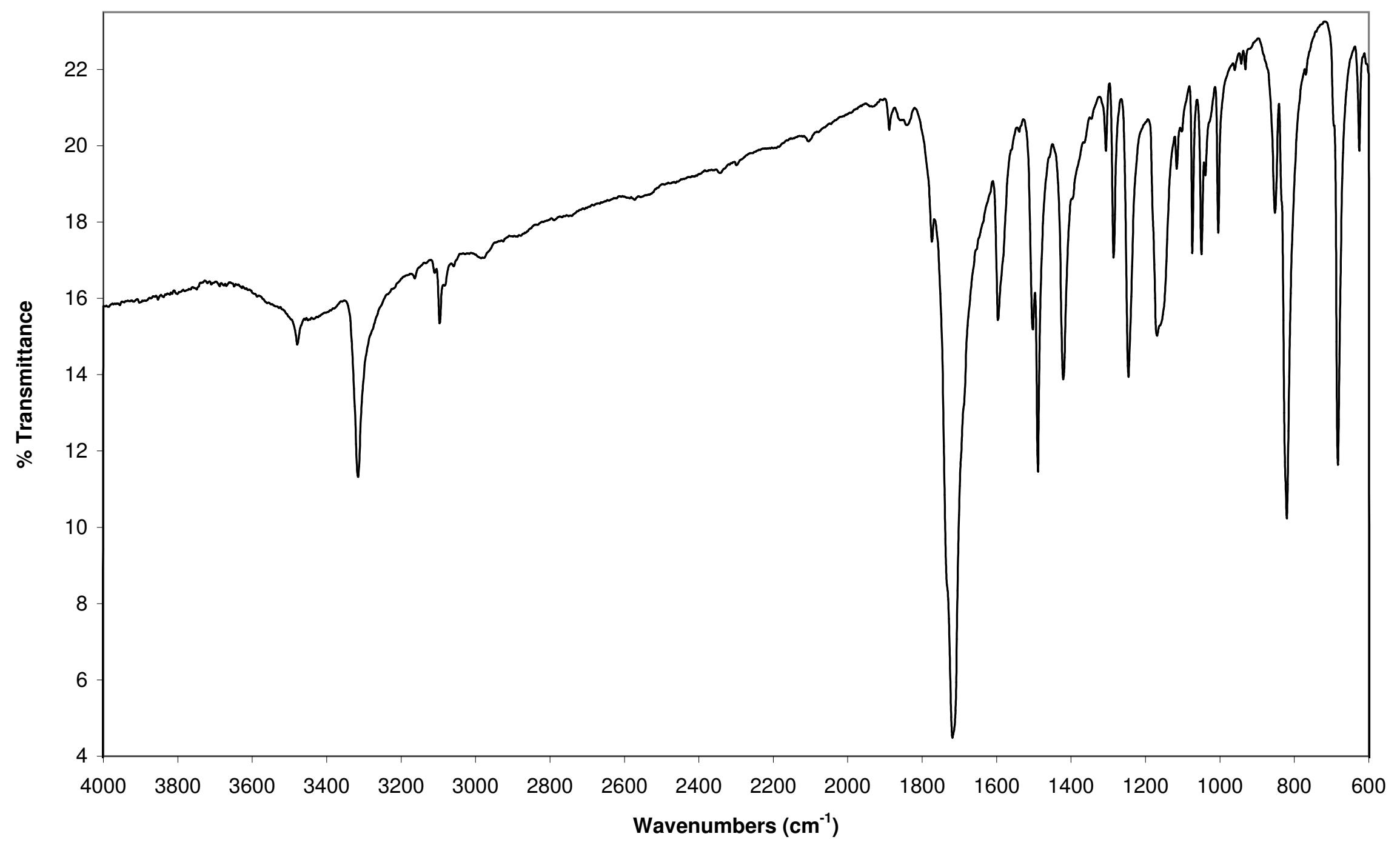




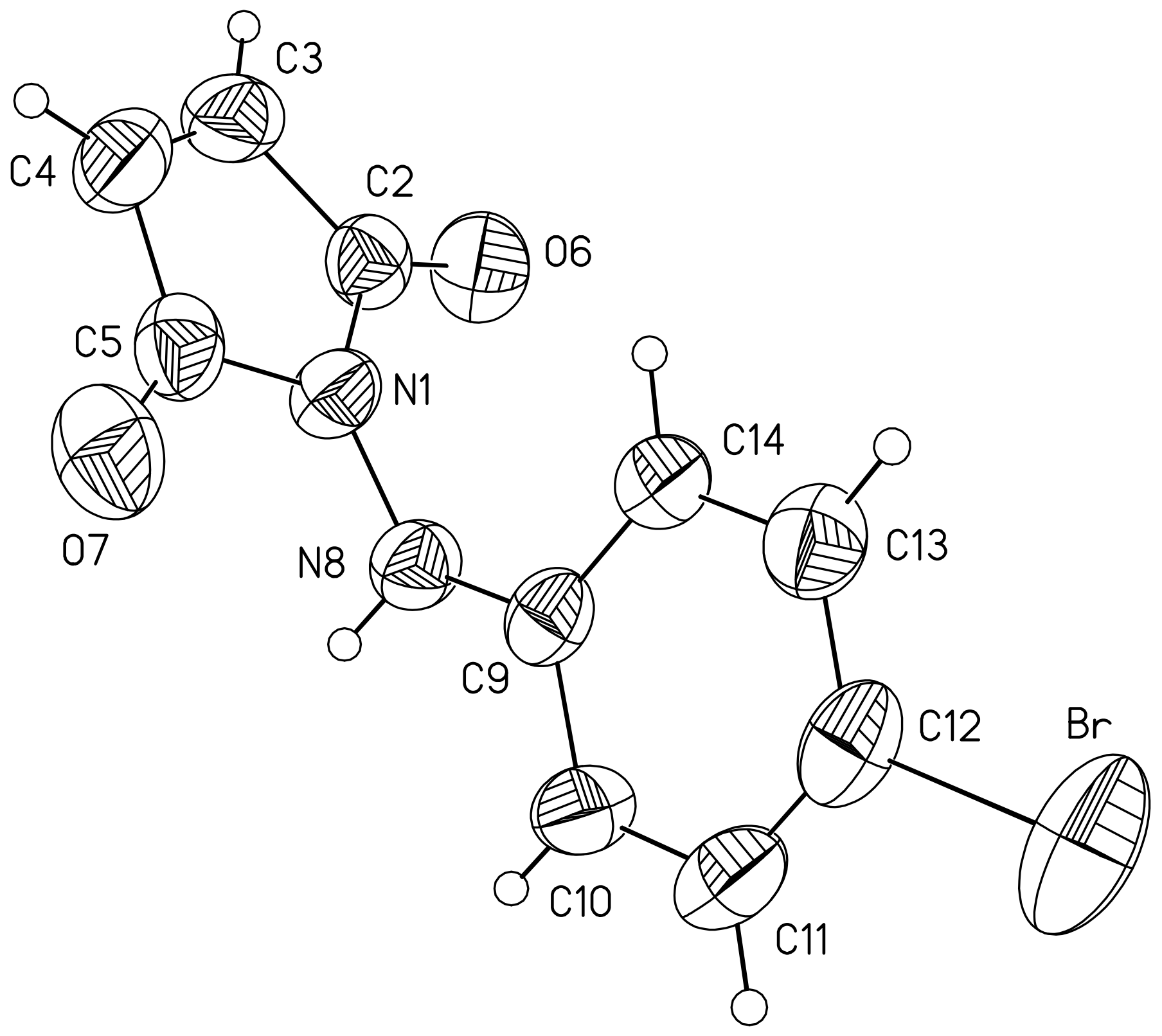

Molecular structure of $\mathbf{2 b}$ showing the atomic labeling scheme with thermal ellipsoids (50\% probability). 


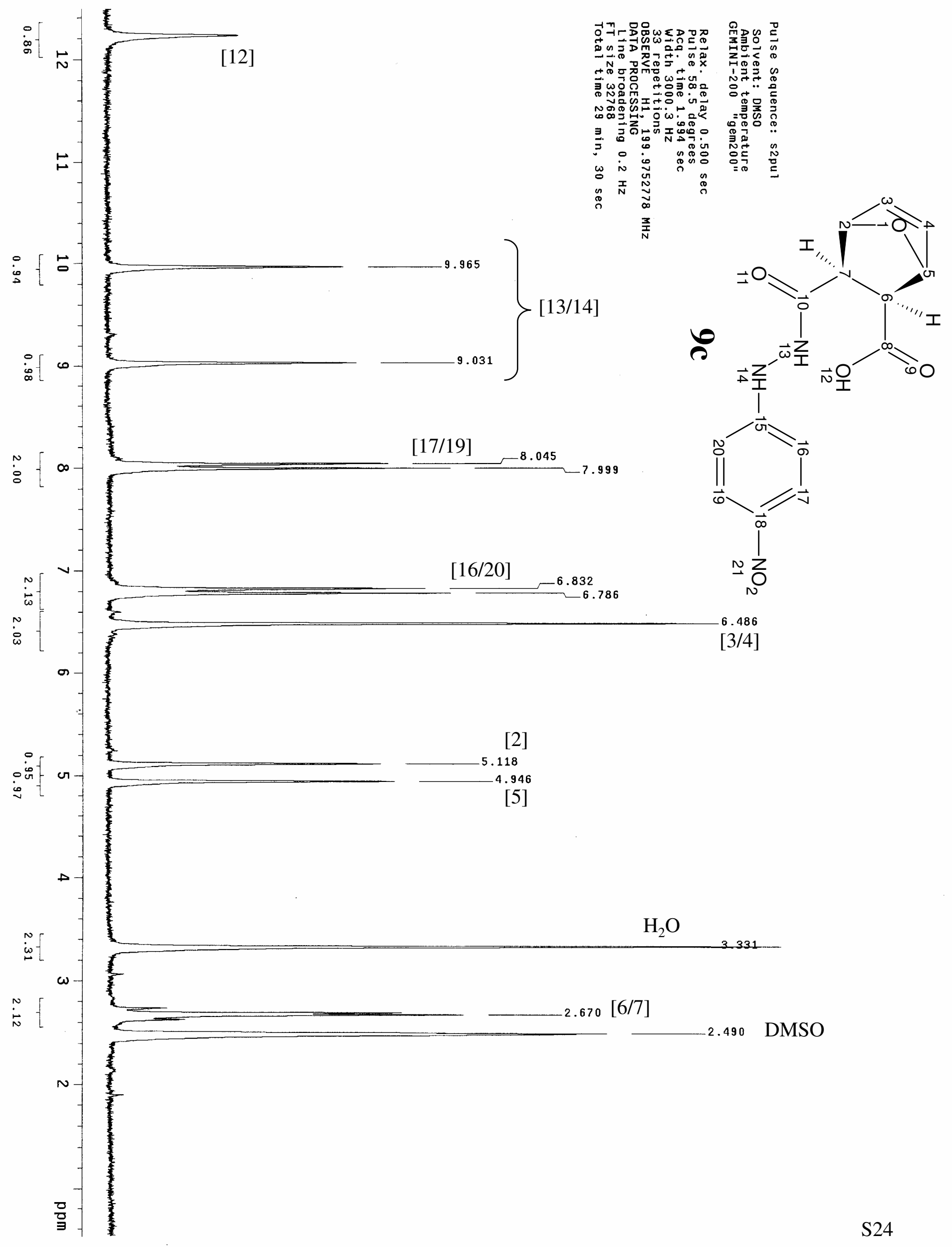




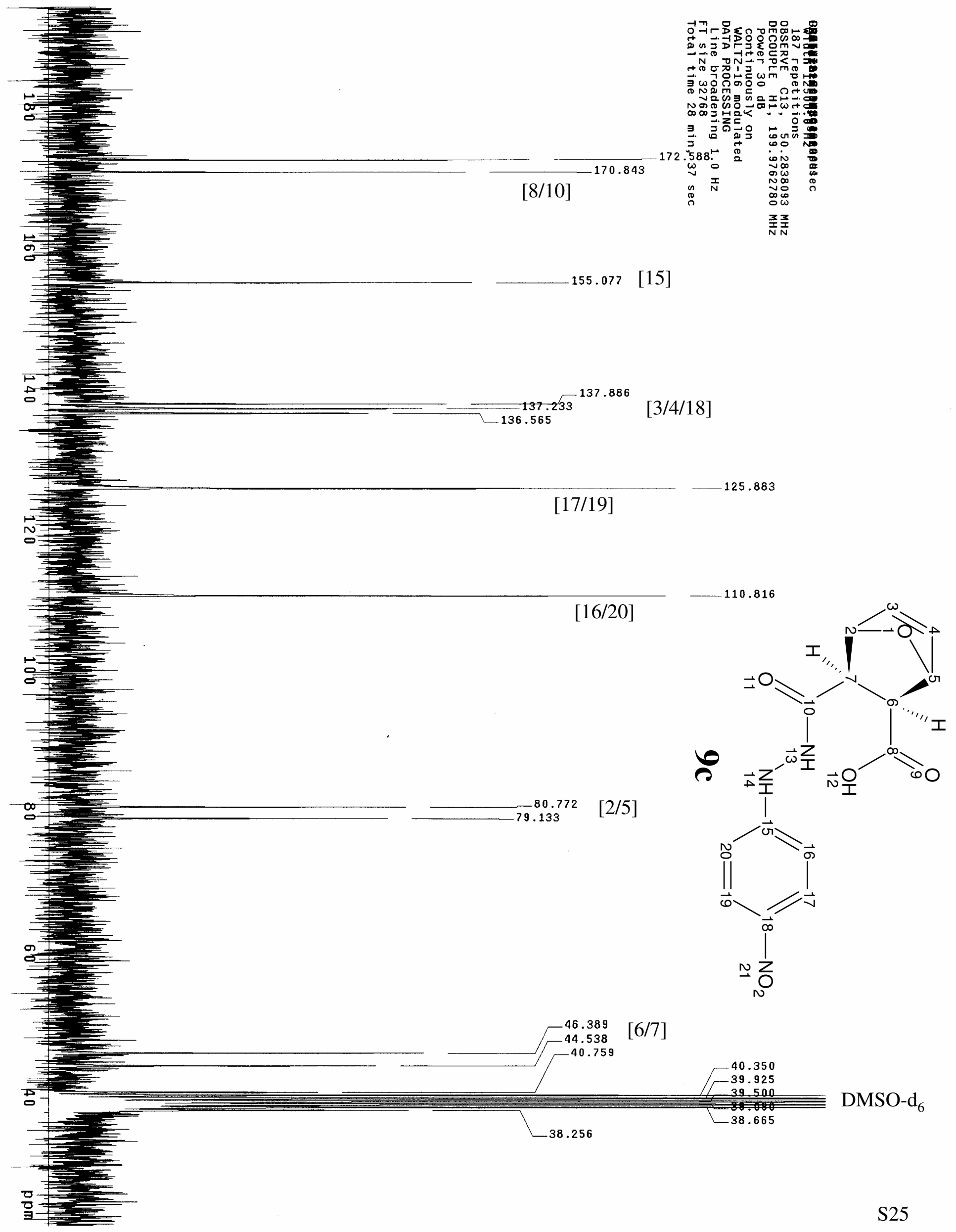




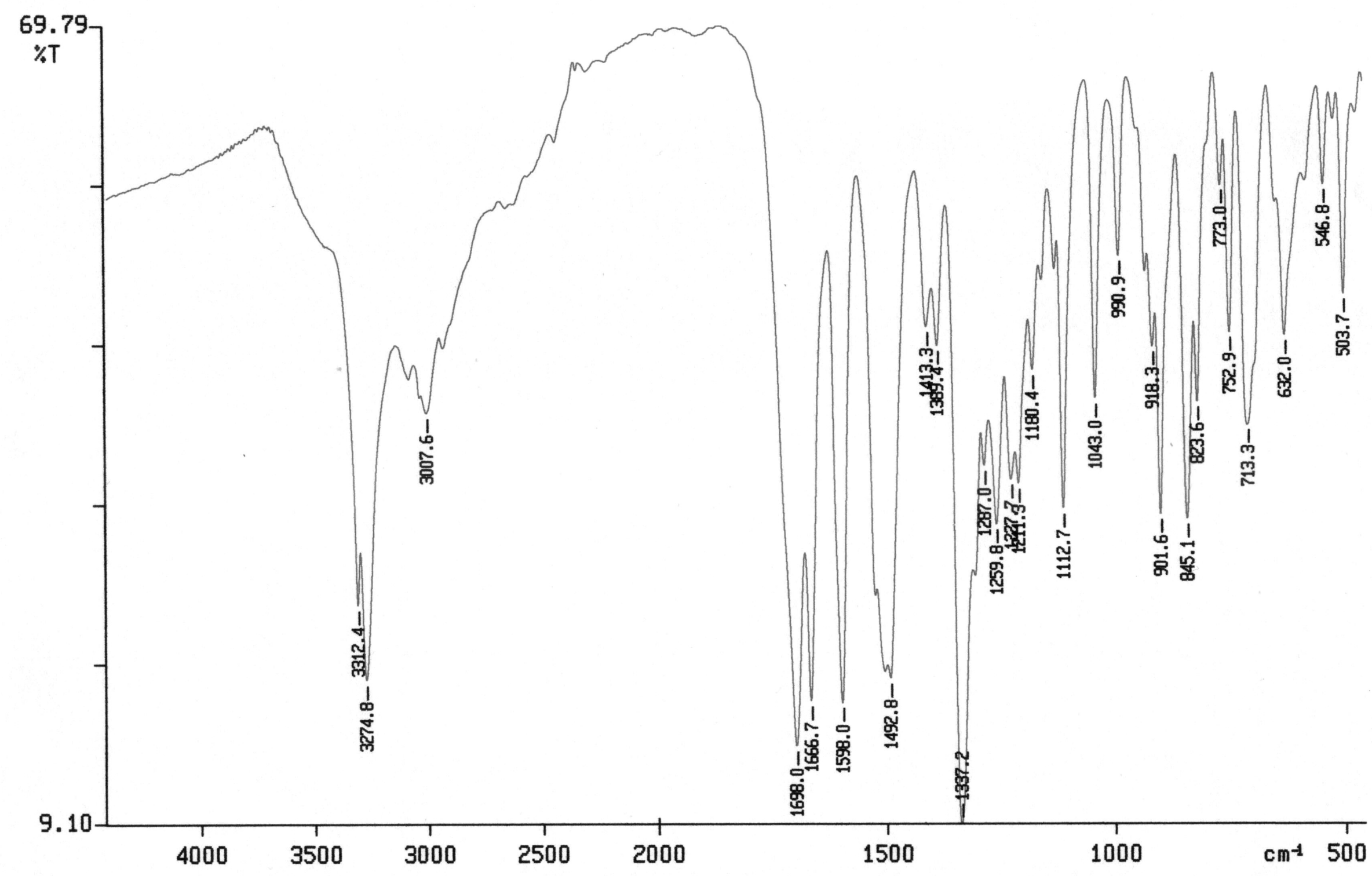

05/03/29 16:43 nick

$X: 4$ scans, $8.0 \mathrm{~cm}-1$ 
Pulse Sequence: s2pur

Solvent: DMSO

Ambient temperature
GEMINI-200 "gem200"

Relax. de lay $0.500 \mathrm{sec}$

Pulse 58.5 degrees

Acq. time 1.994 se

13 repetitions

OBSERVE H1, 199.9752769 MHZ

DATA PROCESSING

Line broadening $0.2 \mathrm{~Hz}$

Total time $2 \mathrm{~min}, 57 \mathrm{sec}$

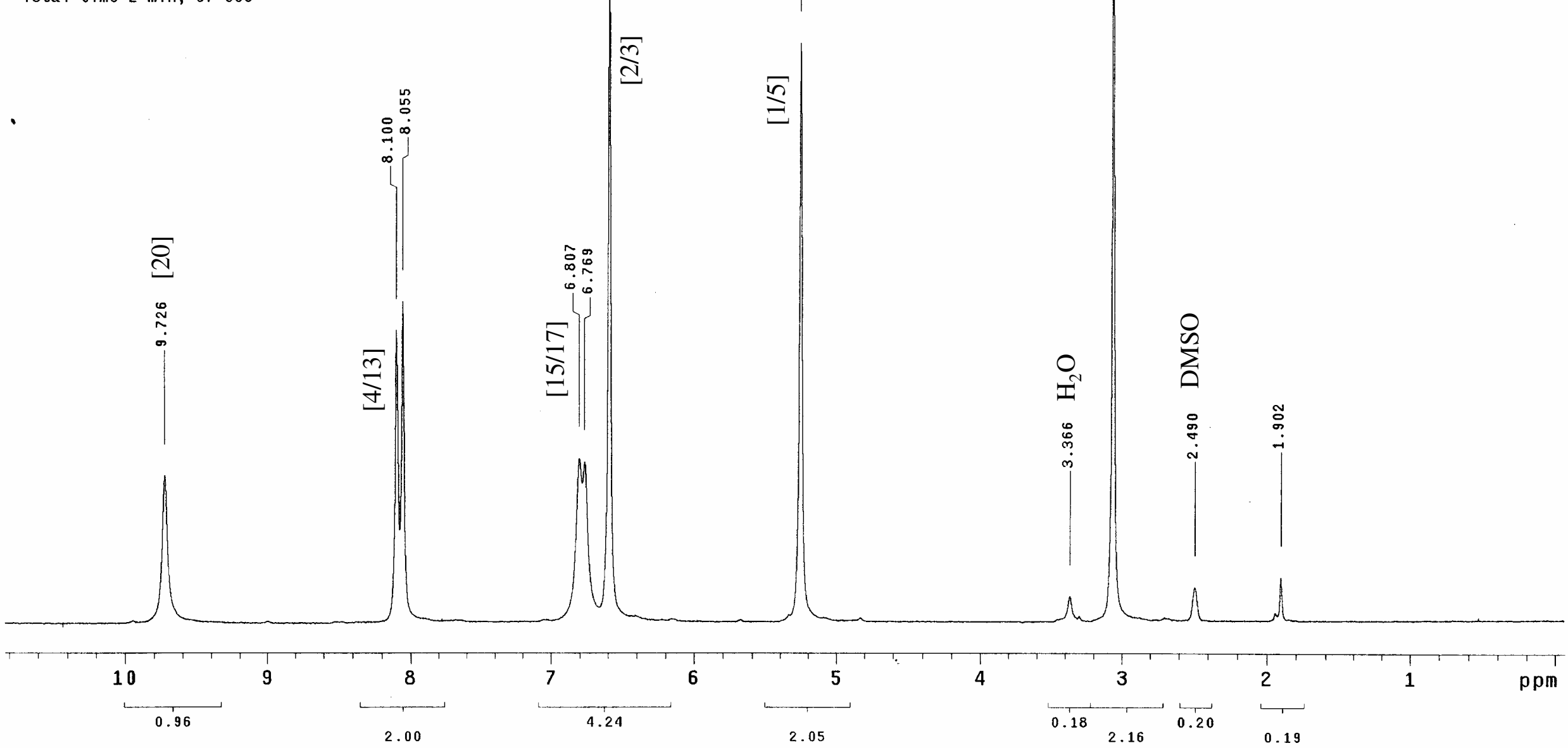




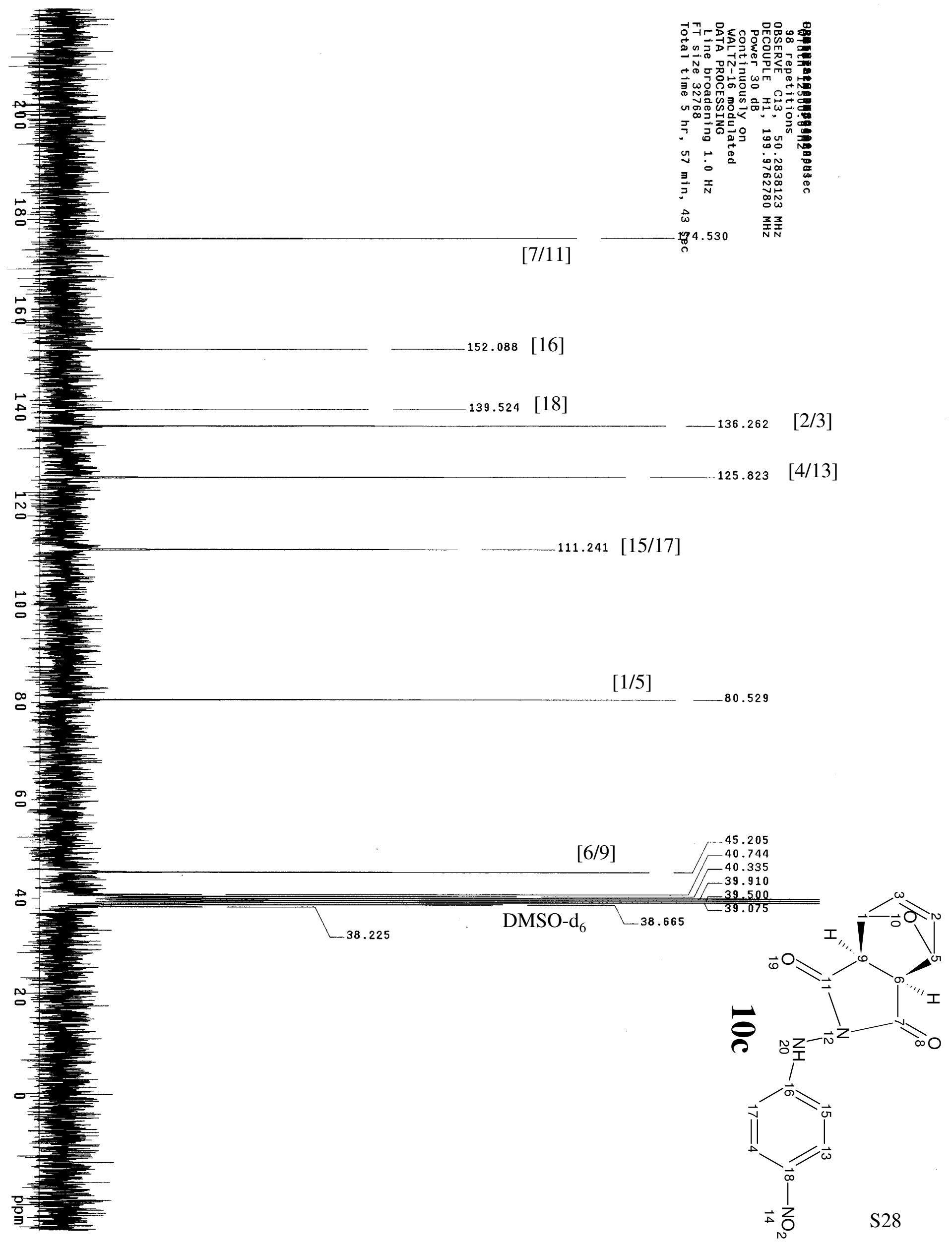




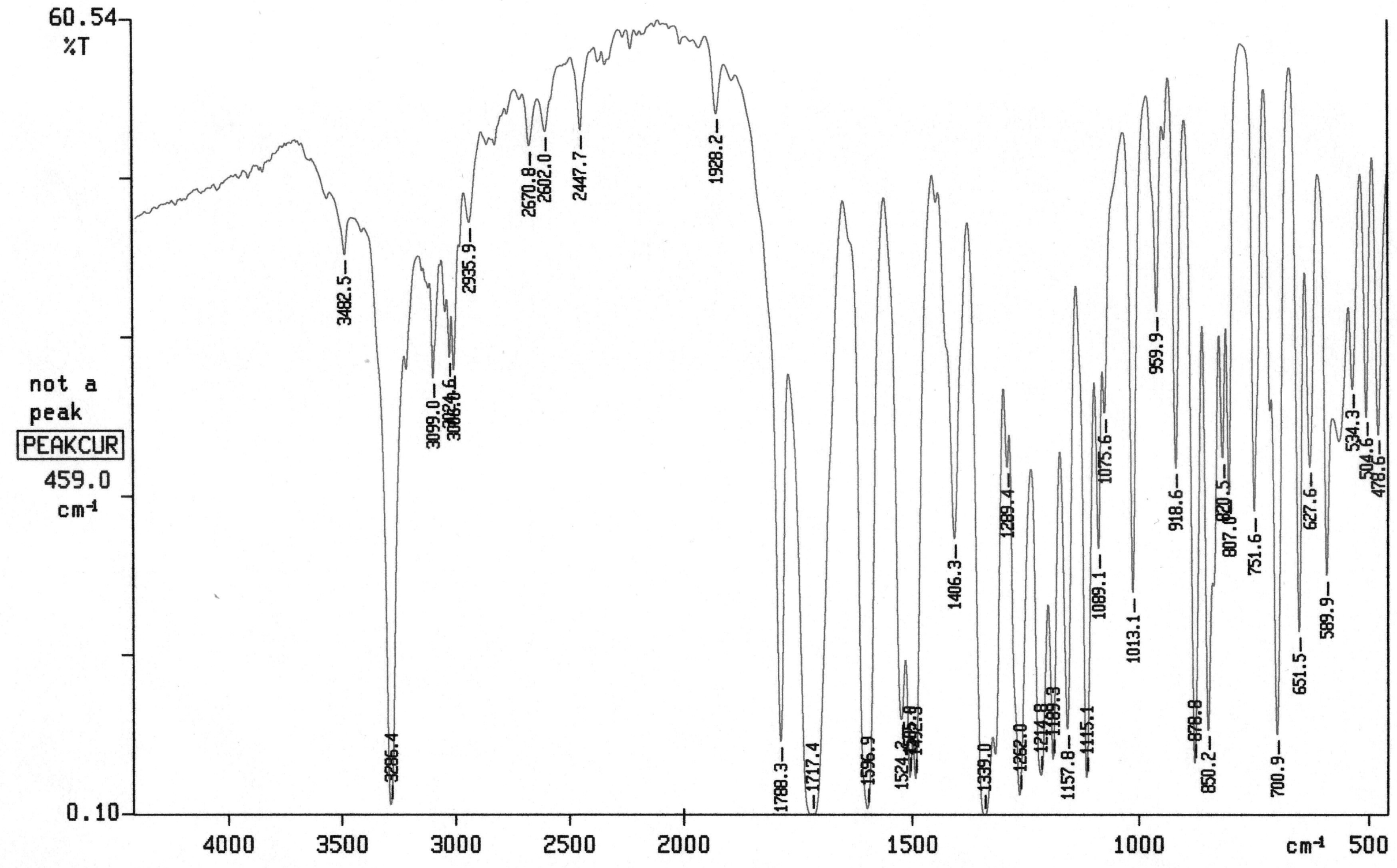

05/03/29 16:55 nick

$X$ : 4 scans, $8.0 \mathrm{~cm}-1$ 


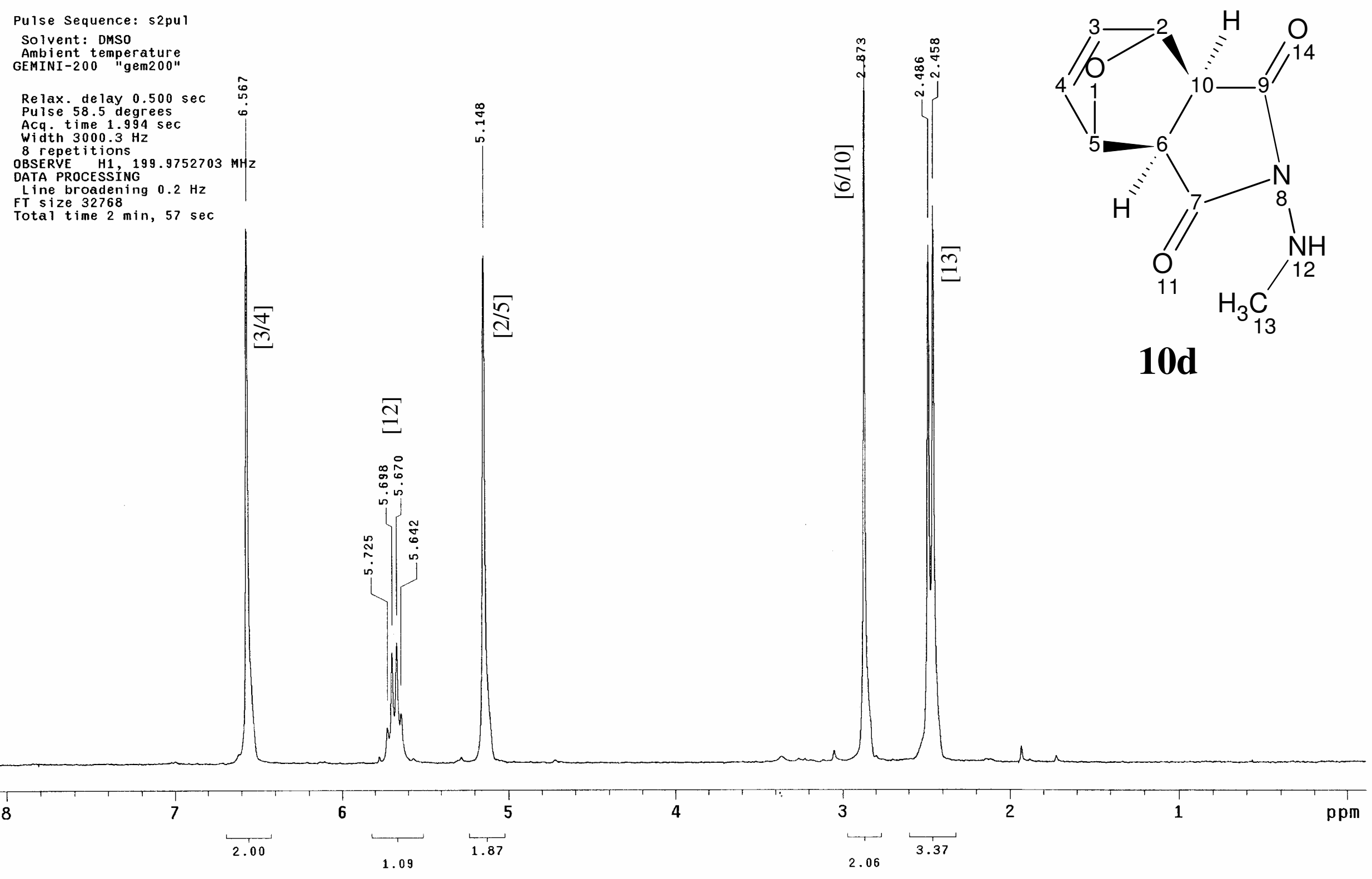




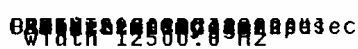

65 repetitions

OBSERVE C13, 50.2835503 MHZ DECOUPLE H1, 199.9753281 MHZ POWer 30 dB

WALTZ-16

DATA PROCESSING

Line broadening $1.0 \mathrm{~Hz}$

Total time $28 \mathrm{~min}, 37 \mathrm{sec}$

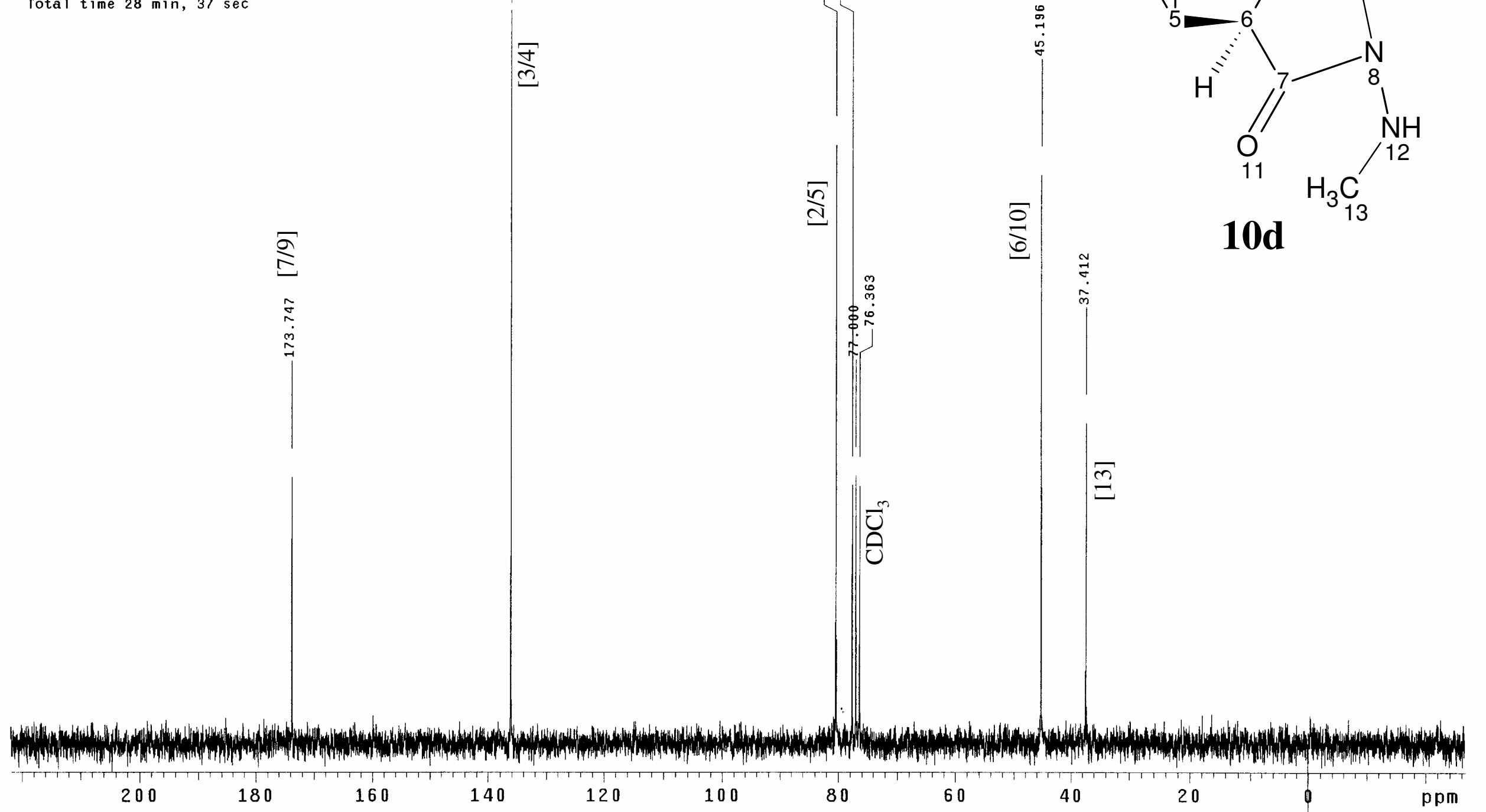


IR (KBr) spectrum of $\mathbf{1 0 d}$

\section{PERKIN ELMER}

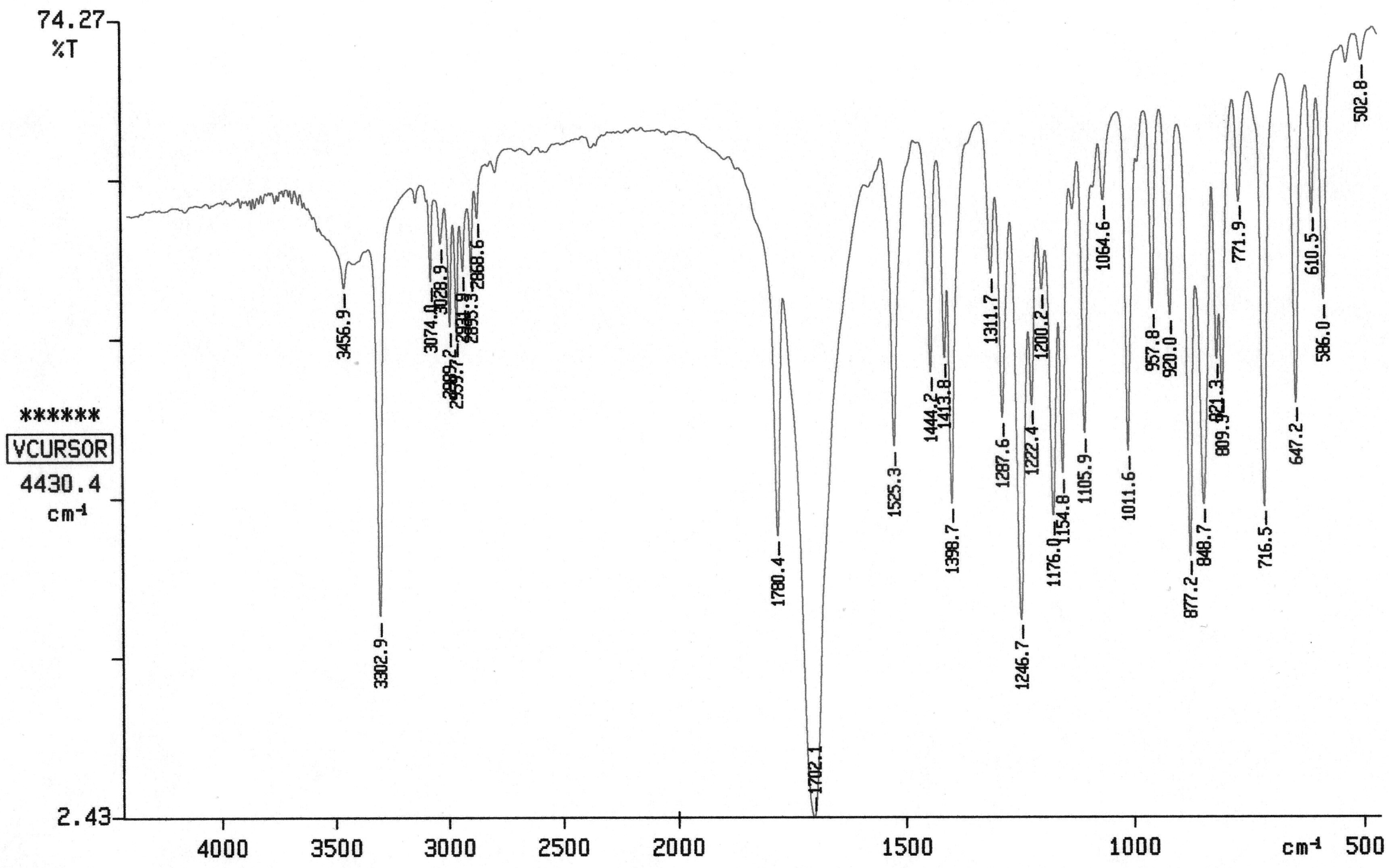

05/03/29 16:15

$X: 4$ scans, $8.0 \mathrm{~cm}-1$ 
Relax. delay $1.000 \mathrm{sec}$ Pulse 15.0 degrees
Acq time 3.813 sec

Acq. time 3.813

64 repetitions

OBSERVE H1, 300.1404558 MHZ

DATA PROCESSING

Line broadening $0.1 \mathrm{~Hz}$

Total time $5 \mathrm{~min}, 8 \mathrm{sec}$
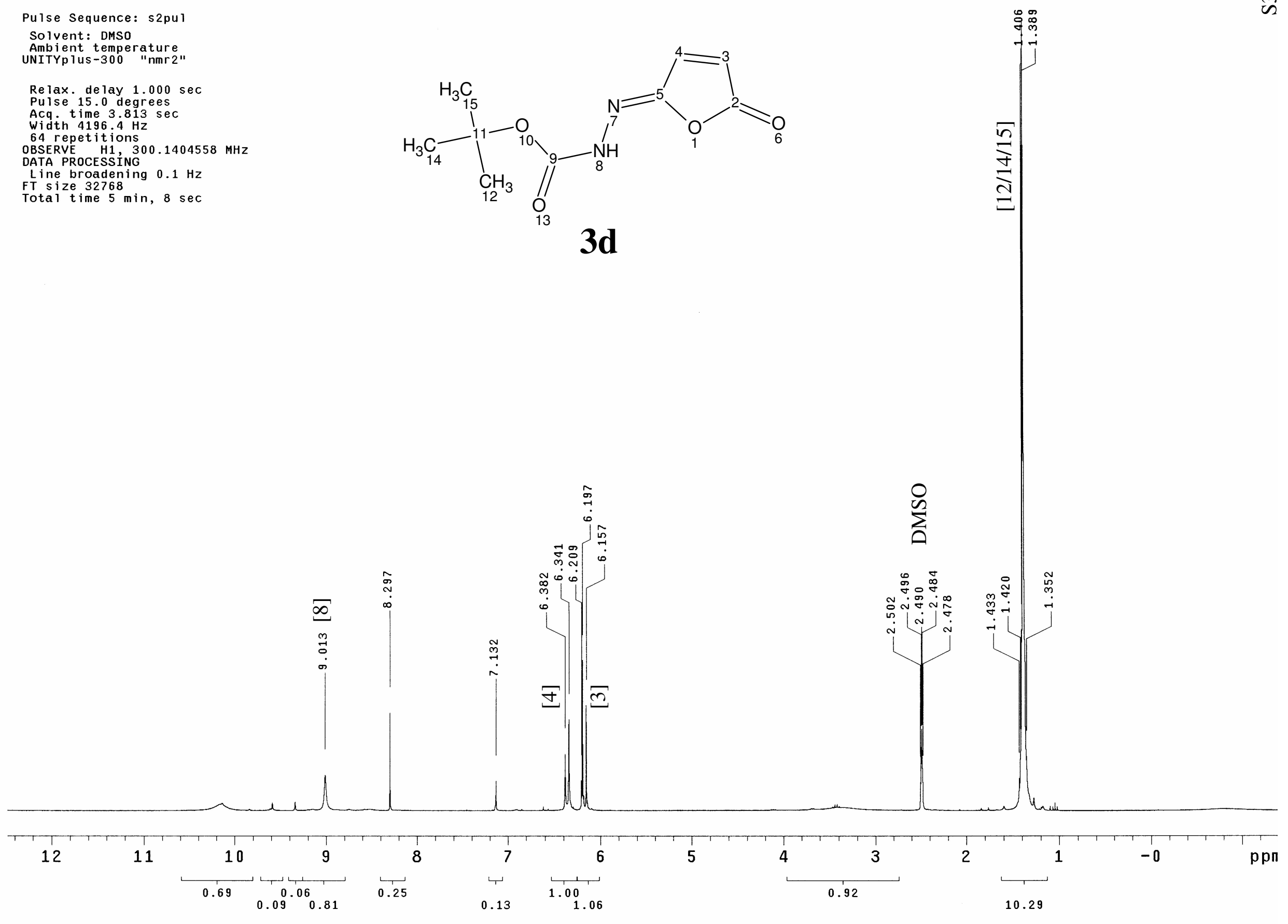


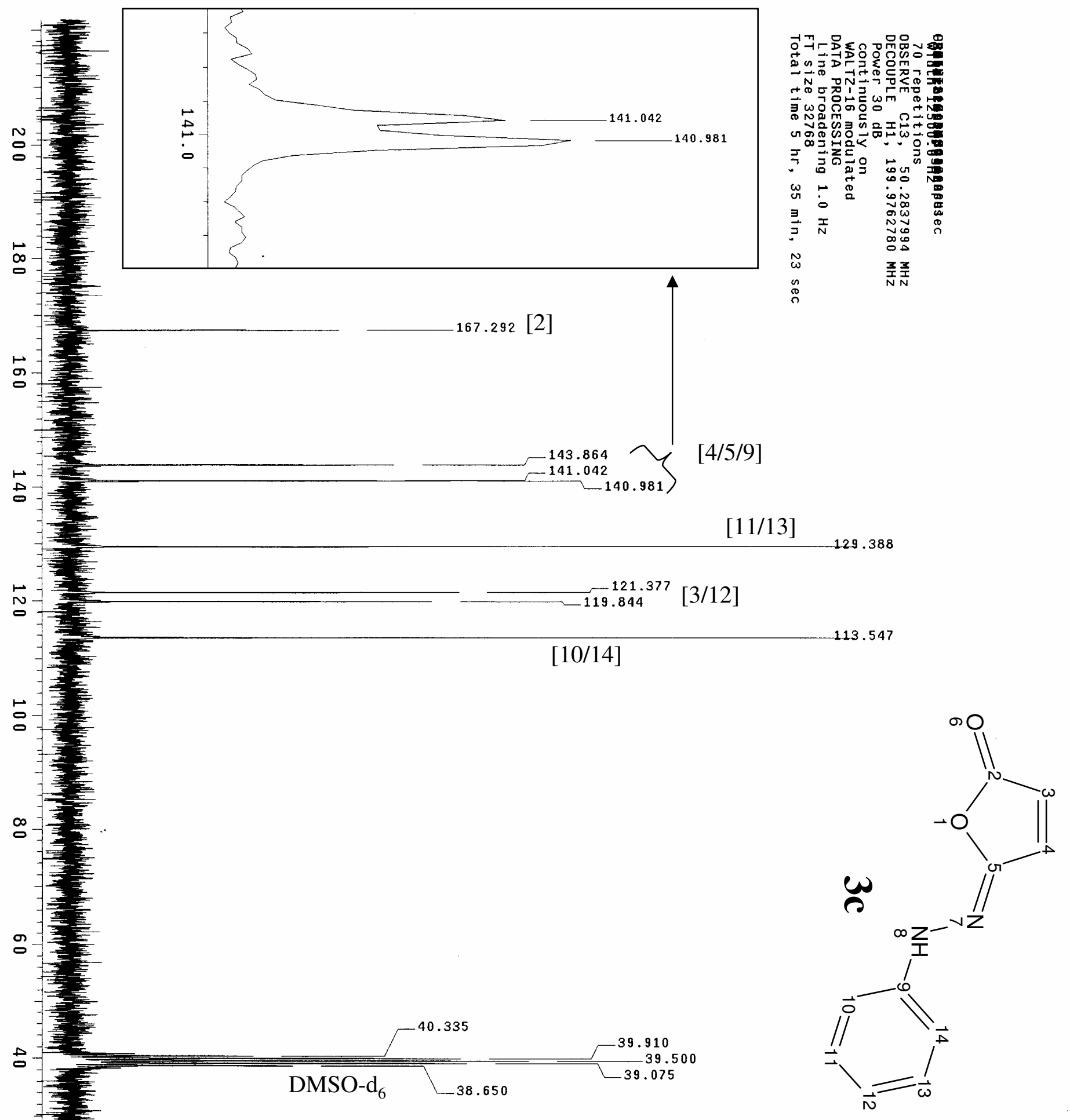

